\title{
The Growth of Galaxies in Cosmological Simulations of Structure Formation
}

\section{Citation}

Murali, Chigurupati, Neal Katz, Lars Hernquist, David H. Weinberg, and Romeel Dave. 2002. "The Growth of Galaxies in Cosmological Simulations of Structure Formation." The Astrophysical Journal 571 (1): 1-14. https://doi.org/10.1086/339876.

\section{Permanent link}

http://nrs.harvard.edu/urn-3:HUL.InstRepos:41381669

\section{Terms of Use}

This article was downloaded from Harvard University's DASH repository, and is made available under the terms and conditions applicable to Other Posted Material, as set forth at http:// nrs.harvard.edu/urn-3:HUL.InstRepos:dash.current.terms-of-use\#LAA

\section{Share Your Story}

The Harvard community has made this article openly available.

Please share how this access benefits you. Submit a story.

Accessibility 


\title{
The growth of galaxies in cosmological simulations of structure formation
}

\author{
Chigurupati Murali, Neal Katz \\ Astronomy Department, University of Massachusetts, Amherst, MA 01003 \\ Lars Hernquist \\ Department of Astronomy, Harvard University, Cambridge, MA 02138 \\ David H. Weinberg \\ Astronomy Department, Ohio State University, Columbus, OH 43210 \\ Romeel Davé \\ Astrophysical Sciences, Princeton University, Princeton, NJ 08544
}

\begin{abstract}
We use hydrodynamic simulations to examine how the baryonic components of galaxies are assembled, focusing on the relative importance of mergers and smooth accretion in the formation of $\sim L_{*}$ systems. In our primary simulation, which models a $\left(50 h^{-1} \mathrm{Mpc}\right)^{3}$ comoving volume of a $\Lambda$-dominated cold dark matter universe, the space density of objects at our (64-particle) baryon mass resolution threshold $M_{c}=5.4 \times 10^{10} M_{\odot}$ corresponds to that of observed galaxies with $L \sim L_{*} / 4$. Galaxies above this threshold gain most of their mass by accretion rather than by mergers. At the redshift of peak mass growth, $z \approx 2$, accretion dominates over merging by about 4:1. The mean accretion rate per galaxy declines from $\sim 40 M_{\odot} \mathrm{yr}^{-1}$ at $z=2$ to $\sim 10 M_{\odot} \mathrm{yr}^{-1}$ at $z=0$, while the merging rate peaks later $(z \approx 1)$ and declines more slowly, so by $z=0$ the ratio is about $2: 1$. We cannot distinguish truly smooth accretion from merging with objects below our mass resolution threshold, but extrapolating our measured mass spectrum of merging objects, $d P / d M \propto M^{-\alpha}$ with $\alpha \sim 1$, implies that sub-resolution mergers would add relatively little mass. The global star formation history in these simulations tracks the mass accretion rate rather than the merger rate. At low redshift, destruction of galaxies by mergers is approximately balanced by the growth of new systems, so the comoving space density of resolved galaxies stays nearly constant despite significant mass evolution at the galaxy-by-galaxy level. The predicted merger rate at $z \lesssim 1$ agrees with recent estimates from close pairs in the CFRS and CNOC2 redshift surveys.
\end{abstract}

Subject headings: cosmology: theory — galaxies: formation 


\section{Introduction}

What galaxies look like today clearly depends on how they were assembled. For example, the formation of ellipticals involves considerably more random kinetic energy than does the assembly of spirals. Thus, it is thought that ellipticals frequently result from the rather violent merger of roughly equal-sized galaxies (Toomre \& Toomre 1972; Barnes \& Hernquist 1992). To investigate this possibility, observational studies have sought to find the merger fraction as a function of redshift and compare this to the present abundance of elliptical galaxies (e.g., Le Fèvre et al. 1999). Conversely, the apparent quiescence of most field spirals implies tight constraints on the amount of merging that can occur in these systems and suggests that these galaxies more likely grew through a process of smooth accretion (e.g., Toth \& Ostriker 1992).

Determining galactic histories and their relation to galaxy properties is the essence of studying galaxy formation. Theoretical investigations of these histories must come to grips with a daunting array of physical processes over a wide range of scales, all of which may play important roles in molding a spectrum of initial density fluctuations into a population of galaxies. Hydrodynamic N-body simulations are a valuable tool because they can represent many of these processes and scales, though they still suffer from finite dynamic range and limited knowledge of the physics of star formation. In principle, one can use such simulations to examine the link between the formation histories and morphological properties of galaxies. Unfortunately, simulations that resolve the internal structure of galaxies and simultaneously model a representative volume of the universe are (slightly) beyond the reach of current computers. Furthermore, the most ambitious efforts to simulate the formation of individual systems do not reproduce the observed distribution of galaxy properties (e.g., Navarro \& Steinmetz 2000), and it is not clear whether this failure has its roots in incorrect cosmological assumptions, an inadequate treatment of physical processes (star formation and feedback in particular), or numerical limitations of the simulations themselves.

In this paper we take a complementary approach to the study of galaxy assembly, using simulations that represent large cosmological volumes but do not resolve the internal structure of galaxies. Within these simulations we measure the global evolution of the number density and total mass of the galaxy population, and we examine the relative importance of smooth accretion and mergers in driving this evolution. We also investigate some related issues such as destruction of galaxies by tidal disruption, mass loss from galaxies during mergers, and the connection between the global star formation history and the history of galaxy assembly. In the context of our adopted cosmological model, these numerical results provide a backdrop for interpreting observations of galaxy evolution and merger rates or constructing theoretical descriptions of the origin of galaxy morphologies. A combination of N-body simulations and analytic methods has led to a fairly complete statistical understanding of the assembly of dark matter halos (see, e.g., Lacey \& Cole 1993; Sheth \& Tormen 2001); our results represent an initial numerical effort to extend that understanding to the baryonic components of galaxies.

This focused examination of the physics of galaxy assembly extends our earlier work on galaxy 
formation in the framework of the inflationary cold dark matter scenario (Katz et al. 1992, 1996, 1999; Hernquist et al. 1995; Weinberg et al. 1997, 1999, 2000). Here we take advantage of the larger simulations possible with the parallel version of TreeSPH (Davé, Dubinski, \& Hernquist 1997). We also take advantage of an emerging consensus, driven by many independent observations, that favors a cosmological model with $\Omega_{m} \approx 0.4, h \equiv H_{0} /\left(100 \mathrm{~km} \mathrm{~s}^{-1} \mathrm{Mpc}^{-1}\right) \approx 0.65$, a flat universe dominated by cold dark matter and vacuum energy, and approximately scale-invariant primeval fluctuations with the properties predicted by inflation. This consensus allows us to concentrate our efforts on a single set of cosmological parameters. However, it is worth bearing in mind that the viability of this model on galactic scales remains a matter of debate (see, e.g., Moore et al. 1999; Spergel \& Steinhardt 2000), and that even within its general framework there are uncertainties in parameter values that could have a significant impact on its predictions.

The principal limitation of our study is that we cannot distinguish the process of truly smooth accretion from mergers with galaxies below our simulations' mass resolution threshold. Throughout this paper, we will use the phrase "smooth accretion" to refer to the combination of these two processes, as distinct from mergers with objects above our resolution threshold. For the large volume, high dynamic range simulation that we rely on for most of our results, the space density of resolved objects implies an identification with galaxies of luminosity $\sim L_{*} / 4$, where $L_{*}$ is the characteristic luminosity in the Schechter (1976) luminosity function (see discussion at the end of $\S 3$.

The following Section describes our numerical simulations and our method of identifying the dense groups of baryonic particles that represent the observable regions of galaxies. In $\S 3$ we investigate the global evolution of the galaxy population - number densities and mass densities - and we discuss numerical resolution issues in some detail. The heart of the paper is $\S 4$, where we examine the processes that create and destroy galaxies in the simulations, the roles of smooth accretion and mergers in galaxy assembly, the statistics of merger masses and mass ratios, mass loss during mergers, and the connection between star formation and accretion. Section 5 discusses the implications of these results, the comparison of our predicted merger rates to observations, and directions for future work. The Appendix presents details of the analysis method, which is based on a view of the simulations as Monte Carlo solutions of a kinetic equation describing the evolution of the galaxy mass function.

\section{Numerical methods}

We perform our simulations using the parallel version of the cosmological N-body/hydrodynamic code TreeSPH (Hernquist \& Katz 1989; Katz, Weinberg, \& Hernquist 1996, hereafter KWH; Davé, Dubinski, \& Hernquist 1997), a code that unites smoothed particle hydrodynamics (SPH) (Lucy 1977; Gingold \& Monaghan 1977) with the hierarchical tree method for computing gravitational forces (Barnes \& Hut 1986; Hernquist 1987). Dark matter, stars, and gas are all represented by particles; collisionless material is influenced only by gravity, while gas is subject to gravitational 
forces, pressure gradients, and shocks. The gas can also cool radiatively, assuming primordial abundances, and through Compton cooling. In $\mathrm{SPH}$, gas properties are computed by averaging or "smoothing" over a fixed number of neighboring particles; the calculations here use 32-particle smoothing. There is a maximum allowed timestep, and all particles are integrated with this step or one a power of two smaller. The timestep criteria are detailed further in KWH and Quinn et al. (1997); we set the tolerance parameter $\eta$ (defined in these papers) to 0.4. In these simulations we include star formation and supernova feedback using the algorithm described in KWH. For each star formation event, supernova energy is added to the surrounding particles as thermal energy on a time scale of $2 \times 10^{7}$ years.

\subsection{Simulation parameters}

All four of the simulations we discuss in this paper are based on a $\Lambda$-dominated cold dark matter $(\mathrm{CDM})$ cosmological model with $\Omega_{m}=0.4, \Omega_{\Lambda}=0.6, h \equiv H_{0} /\left(100 \mathrm{~km} \mathrm{~s}^{-1} \mathrm{Mpc}^{-1}\right)=0.65$, and primeval spectral index $n=0.93$. With the tensor mode contribution, normalizing to COBE using CMBFAST (Seljak \& Zaldarriaga 1996; Zaldarriaga, Seljak, \& Bertschinger 1998) implies a normalization $\sigma_{8}=0.8$, which provides a good match to cluster abundances (White, Efstathiou, \& Frenk 1993). We use the $\mathrm{Hu} \&$ Sugiyama (1996) formulation of the transfer function, and we adopt a baryonic density $\Omega_{b}=0.02 h^{-2}$, consistent with the deuterium abundance in high redshift Lyman limit systems (Burles \& Tytler 1997, 1998), and the opacity of the high redshift Lyman-alpha forest (e.g. Rauch et al. 1997). All of our simulations model a triply periodic cubical volume.

The simulations cover a range of resolutions, which allows us to examine the numerical behavior and test for convergence. The primary and largest simulation, L50/144 (Davé et al. 2001), has 144 gas and dark matter particles in a comoving periodic box $50 h^{-1} \mathrm{Mpc}$ on a side, with a gravitational softening length $\epsilon_{\text {grav }}=7 h^{-1}$ comoving kpc (equivalent Plummer softening). This simulation is evolved to $z=0$. As discussed in $\S 2.3$ below, we adopt as our nominal gas mass resolution the mass corresponding to $64 \mathrm{SPH}$ particles, which for this simulation is $5.4 \times 10^{10} M_{\odot}$. We also analyze L11/64, a simulation with $64^{3}$ gas and dark matter particles in a volume $11.11 h^{-1} \mathrm{Mpc}$ on a side, also evolved to $z=0$. This simulation has a particle mass eight times smaller than L50/144 and a gravitational softening length two times smaller. The minimum SPH smoothing length is $\epsilon_{\text {grav }} / 4$, so the higher spatial resolution extends to the hydrodynamic forces. We also analyze L11/128, a simulation with $128^{3}$ particles of each type in a volume the same size as the L11/64 simulation, but only evolved to $z=3$. It has a particle mass eight times smaller than L11/64 and a gravitational softening length two times smaller. Finally, we analyze L11/64'. This simulation has the same resolution and volume as L11/64, but it uses the same random realization of the CDM power spectrum as L11/128. It has been evolved to $z=3$, and the comparison to L11/128 gives our most direct test of finite resolution effects down to this redshift.

Our results depend on the spatial and mass resolution of the simulation, which we discuss in

more detail below, and on the temporal spacing of the available outputs. Large time intervals tend 
to increase the amount of smooth accretion because small groups can form and merge with larger neighbors within a single time interval. For our analysis, we chose a sequence of outputs with a spacing of $\Delta z=0.5$ for $7 \geq z \geq 4, \Delta z=0.25$ for $4 \geq z \geq 1$, and $\Delta z=0.125$ for $1 \geq z \geq 0$. The corresponding time intervals, as seen in the figures below, are roughly $1 / 3 \mathrm{Gyr}, 2 / 3 \mathrm{Gyr}$ and $4 / 3 \mathrm{Gyr}$, respectively. These are on the order of the infall time scale at the corresponding epoch, although perhaps somewhat longer at the latest time. We repeated some of our main analyses using twice as many outputs, i.e. halving the redshift spacing, and found similar results.

\subsection{Group finding algorithm}

SPH simulations with CDM initial conditions and radiative cooling lead to the formation of dense groups of baryonic particles that have sizes and masses comparable to the luminous regions of observed galaxies (Katz et al. 1992; Evrard, Summers, \& Davis 1994). If star formation is included, these dense groups are the regions where stars form (KWH). The accretion and merger histories of these objects are the subject of this paper, so the identification of distinct particle groups underlies all of our subsequent analysis. We use the group finding algorithm of Stadel et al. (2001), Spline Kernel Interpolative DENMAX, abbreviated SKID (Gelb \& Bertschinger 1994; see also KWH and http://www-hpcc.astro.washington.edu/TSEGA/tools/skid.html). The basic algorithm consists of: 1) determining the smoothed density field; 2) moving particles upward along the gradient of the density field using an heuristic equation of motion that forces them to collect at local density maxima; 3) defining the approximate group to be the set of particles that aggregate at a particular density peak; 4) finally, removing particles from the group that do not satisfy a negative energy binding criterion relative to the group's center of mass. ${ }^{1}$ In contrast to the identification of dark matter halos in N-body simulations, there is essentially no ambiguity about the identification of

\footnotetext{
${ }^{1}$ This procedure starts with the highest energy particles and updates the potential as particles are lost.
}

Table 1: Simulations

\begin{tabular}{ccccccc}
\hline Name & $L(\mathrm{Mpc})$ & $N$ & $z_{\text {fin }}$ & $M_{\max }\left(M_{\odot}\right)^{\mathrm{a}}$ & $M_{\min }\left(M_{\odot}\right)^{\mathrm{b}}$ & $M_{c}\left(M_{\odot}\right)^{\mathrm{c}}$ \\
\hline L50/144 & $50 h^{-1}$ & $2 \times 144^{3}$ & 0 & $3.1 \times 10^{12}$ & $6.8 \times 10^{9}$ & $5.4 \times 10^{10}$ \\
L11/64 & $11 h^{-1}$ & $2 \times 64^{3}$ & 0 & $3.2 \times 10^{10}$ & $8.5 \times 10^{8}$ & $6.8 \times 10^{9}$ \\
L11/128 & $11 h^{-1}$ & $2 \times 128^{3}$ & 3 & $3.0 \times 10^{10}$ & $1.1 \times 10^{8}$ & $8.5 \times 10^{8}$ \\
L11/64 & $11 h^{-1}$ & $2 \times 64^{3}$ & 3 & $3.0 \times 10^{10}$ & $8.5 \times 10^{8}$ & $6.8 \times 10^{9}$ \\
\hline
\end{tabular}

\footnotetext{
${ }^{a}$ Mass of $10^{\text {th }}$ largest galaxy in simulation, at $z=0$ for L50/144 and $z=3$ for other simulations

${ }^{b}$ Mass of 8-particle group

${ }^{c}$ Mass of 64-particle group
} 
distinct baryon clumps in these SPH simulations because dissipation greatly increases the density of cooled baryons with respect to the local background.

As output, SKID produces a list of baryonic particles, both gas and stars, that belong to each identified group. To be included in a group, gas particles must have a temperature $T<3 \times 10^{4} \mathrm{~K}$ and and a density $\rho>10^{3} \Omega_{b}$. In effect, we consider only the cold gas and stars, the material that comprises the bulk of the matter in the central, visible regions of galaxies. Therefore, throughout this paper we use the terms group and galaxy interchangeably.

One complication that arises with this group definition is lost particles. Specifically, given all the particles in groups at one output, SKID finds that roughly $1 \%$ of these are lost by the next output. However, more careful inspection shows that roughly $30 \%$ of those particles declared lost still reside physically within the group boundary, which we define to be the maximum radius of any bound member particle. These particles may in fact be unbound and in the process of leaving the group, although we have not checked their energies relative to the group center-ofmass. Nevertheless, in the interest of estimating the mass loss conservatively, we simply add these particles back to the group. Consequently, our group definitions become dependent on output spacing, although the effect is only of order $1 \%$.

\subsection{Mass resolution}

We allow SKID to identify groups with as few as eight particles. Because these particles must be stars or cooled baryons, even these small groups are real, high contrast objects residing in dense backgrounds. However, our comparisons, statistical and direct, between simulations with different mass resolutions show that a simulation's group list becomes incomplete (relative to a simulation with higher resolution) below $\sim 60$ particles. Above this threshold, the total baryon mass of groups is well converged (see the discussion of Figure 1 below), though the division into stellar and gas components remains resolution dependent up to a somewhat higher threshold. For most of the analyses of this paper, we adopt $N_{g r p}=64$ particles as the minimum number for a resolved group, and we discard all SKID groups with fewer particles. Our nominal mass resolution limit is therefore that of $64 \mathrm{SPH}$ particles, though since mass is redistributed among particles during star formation (see KWH) the exact mass of a 64-particle group may be slightly higher or lower. We further discuss resolution effects and other limits on the mass ranges probed by our simulations in the next Section.

\section{Evolution of galaxy number and mass densities}

First we look at the simplest quantities that characterize the galaxy populations in the simulations: the mass function, the total number density, and the total mass density. Examining these characteristics shows qualitatively how the galaxy population evolves and also illustrates the 
numerical factors that come into play in the analysis.

As mentioned above, the most important numerical variable is the mass resolution, the minimum mass of a resolved particle group. Because the number of galaxies increases towards low masses, the number of galaxies in a simulation depends sensitively on this lower mass limit. The box size also comes into play, however, as this determines the number of relatively rare, highmass objects that form, thereby determining the maximum mass for which statistical results are meaningful.

Table 1 lists the numerical parameters of the simulations described in $\S 2.1$, in particular the characteristic minimum and maximum masses associated with the resolution and box size. We define the maximum mass, $M_{\max }$, to be the baryonic mass of the $10^{\text {th }}$ largest galaxy in the simulation volume, identified as described in $\S 2.2 . M_{\min }$ denotes the mass corresponding to an 8-particle minimum group size, and $M_{c}$ denotes the adopted cutoff mass corresponding to the 64-particle minimum. All three $11.11 h^{-1}$ volumes have about the same maximum mass at $z=3$. The minimum mass is determined by the resolution, so the L11/128 simulation has a substantially lower minimum mass.

Figure 1 shows particle groups in the L50/144, L11/64, and L11/128 simulations at $z=3$. In any given simulation, the mass function begins to turn over at a mass 5-10 times higher than the 8-particle minimum of the SKID groups. Comparison to the next higher resolution simulation shows that this turnover is a numerical artifact. If we instead restrict attention to masses above our adopted 64-particle threshold $M_{c}$, then the mass function in a higher resolution simulation gives a fairly smooth continuation of that in the lower resolution simulation. The envelope of the three mass functions approximately follows a Schechter function.

All the analyses that follow include only galaxies with masses above $M_{c}$. Because of their different resolutions and box sizes, our simulations probe different mass ranges. In the case of L50/144, one can see from Figure 1 that this range is nearly disjoint from that of the smaller volume simulations.

The lower panel of Figure 2 compares the comoving number density $\nu$ of galaxies above the 64particle threshold in the four simulations, as a function of time (lower axis label) or redshift (upper axis label). The upper panel shows the comoving density $\mu$ of baryonic mass in these resolved galaxies. Once again, the effects of mass resolution and box size are clearly evident. L11/128 has the highest number and mass densities. L11/64 and L11/64' have fewer galaxies, of course, since they have the same upper mass cutoff as L11/128 but a lower mass cutoff that is eight times higher. However, if the power law form of the low end of the mass function, roughly $n(M) \propto M^{-1.1}$ in Figure 1, continued to arbitrarily high mass, then L50/144 would have the highest number and mass densities because it has the largest dynamic range $M_{\max } / M_{c}$. (For $n \propto M^{-1}$, the number of galaxies per logarithmic interval is constant, and the mass per logarithmic interval increases linearly with $M$.) In reality, the maximum mass $M_{\max }$ of L50/144 lies well into the exponential cutoff regime of the mass function, and as a consequence the galaxy population of L50/144 has the 


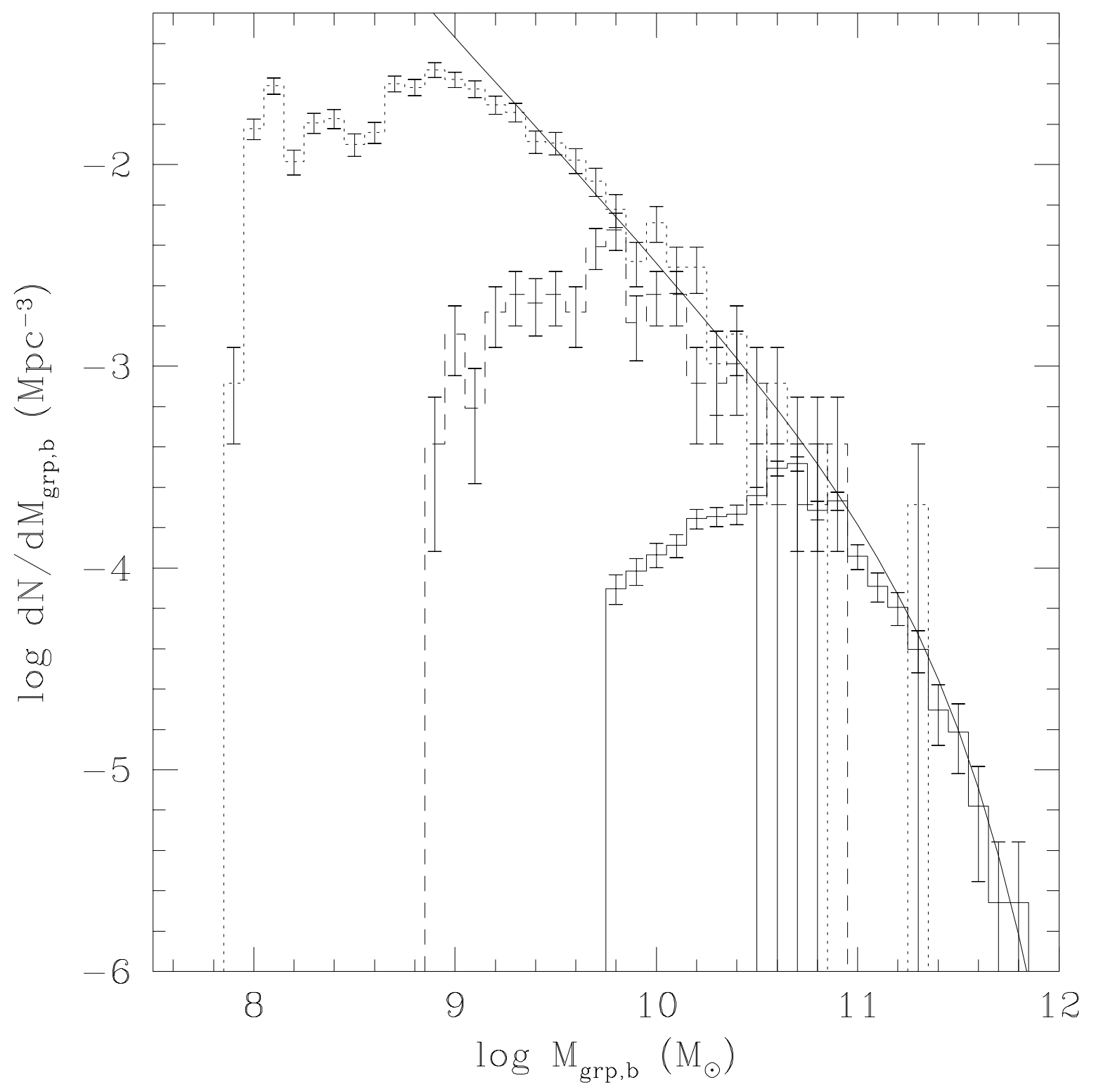

Fig. 1. - The mass function at $z=3$ in the L50/144 (solid histogram), L11/64 (dashed histogram), and L11/128 simulations (dotted histogram). Error bars reflect Poisson errors. The solid curve is a Schechter function overlaid for comparison. 
lowest mass density and by far the lowest number density of the four simulations.

Turning from numerical considerations to evolution, we see that number and mass densities both increase most strongly at early times, i.e. for $z>2$. In the simulations that continue to $z=0$, the number of objects becomes approximately constant for $z<2$, and the difference in mass density between L50/144 and L11/64 stays about the same. As discussed in $\S 4$ below, the number of galaxies remains roughly constant even though merging continues to late times because new galaxies also continue to form. In contrast to the number density, the total mass in galaxies continues to grow, both from the formation of new groups and from the accretion fed growth of existing galaxies.

Figure 3 shows this behavior in terms of the corresponding rates of change in mass density $\dot{\mu}$ and number density $\dot{\nu}$. The differences between simulations are greatest at high redshift, where the hierarchical mass scale is lowest. For $z<2$ the results at different resolutions agree, indicating that the formation rate of very low mass objects has become negligible. The differences in total mass and number density apparent in Figure 2 must therefore be imprinted at early times.

The physical quantity one would like to know is the mass or number density of galaxies above some specified mass $M$. In practice $M$ cannot be below the mass resolution of the simulation, $M_{c}$, and the mass and number densities measured by the simulations are not those for all galaxies above a given mass but only for galaxies below a maximum mass determined by the volume. However, we can measure the same quantity in more than one of our simulations to assess the robustness of our measurements given these limitations. The L11/64 and L11/64' simulations have the same resolution and box size, but there is a small difference in the quantities plotted in Figures 2 and 3, represented by the filled squares and four point stars. These differences show the random fluctuations associated with two different realizations of the initial power spectrum in this volume. We can assess the impact of our finite resolution on these quantities by comparing the L11/64' simulation (four point stars) to the L11/128 simulation analyzed with the mass cutoff $M_{c}=6.8 \times 10^{9} M_{\odot}$ of L11/64'. These results are shown by the three point stars in Figures 2 and 3 . The good agreement between the three and four point stars indicates that we can robustly measure number and mass densities for galaxies above the 64-particle threshold mass $M_{c}$, and that finite resolution effects on our statistics above this threshold should be small.

To relate our numerical results to an observational context, we would like to know the approximate luminosity of galaxies corresponding to our resolution threshold $M_{c}$. Although we have stellar masses for each of our galaxies, the mass-to-light ratio of the stellar populations depends sensitively on the assumed initial mass function and on the age, metallicity, and extinction of the stellar population. Furthermore, the overall scale of the galaxy baryon masses may be sensitive to some cosmological parameters, especially $\Omega_{b}$. Given these uncertainties, the most robust way to identify our mass threshold with an approximate luminosity threshold is by matching the space densities of simulated and observed galaxy populations. If we match the mass function in Figure 1 to Blanton et al.'s (2001) determination of the luminosity function from the Sloan Digital Sky Sur- 


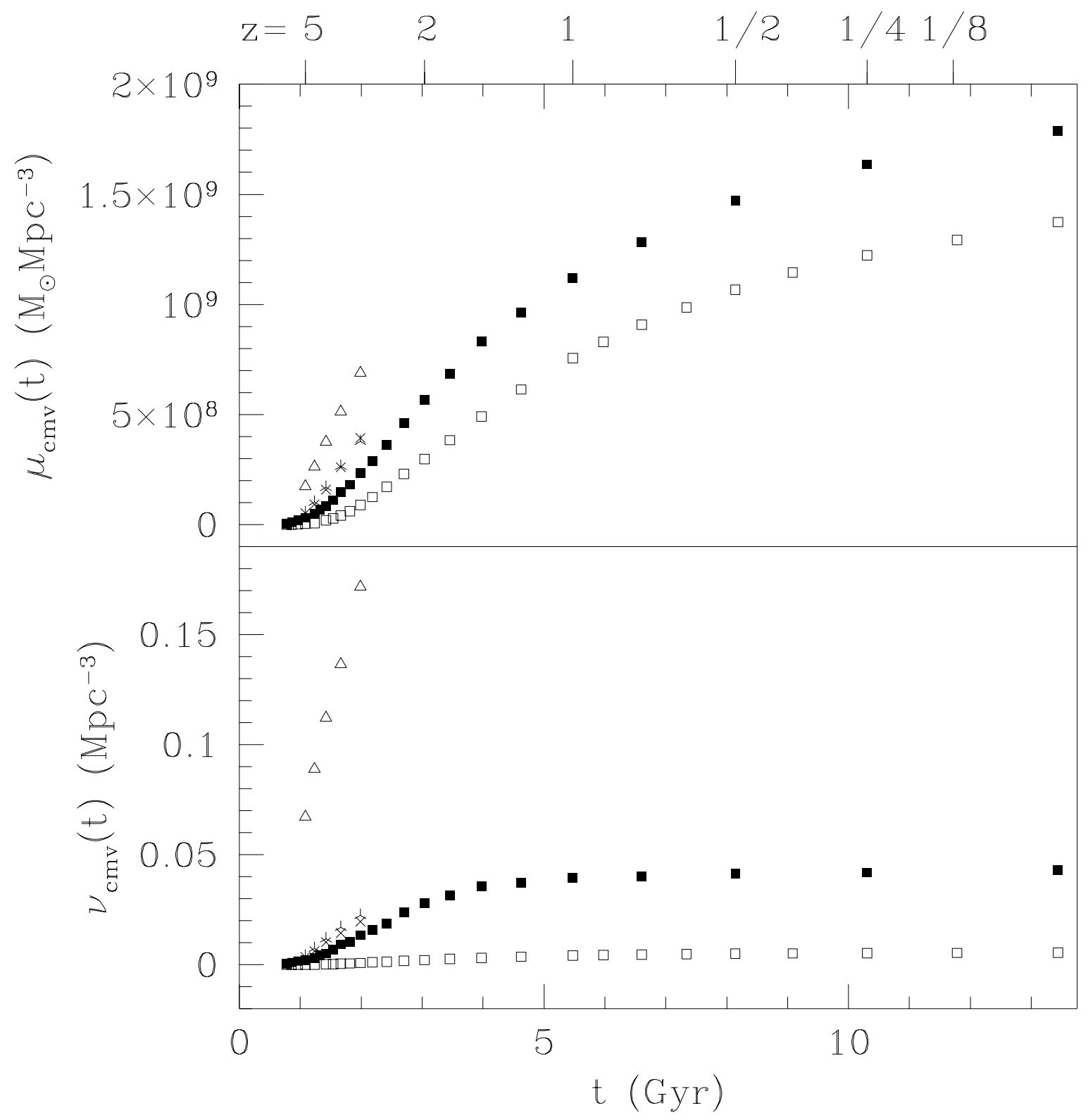

Fig. 2.- The total comoving mass (top) and number density (bottom) of galaxies as a function of cosmic time for galaxies with masses above $M_{c}=5.4 \times 10^{10} M_{\odot}$ (L50/144-open squares), $M_{c}=$ $6.8 \times 10^{9} M_{\odot}(\mathrm{L} 11 / 64$-solid squares, L11/64'-four point stars, L11/128-three point stars), and $M_{c}=8.5 \times 10^{8} M_{\odot}(\mathrm{L} 11 / 128-$ open triangles $)$. 


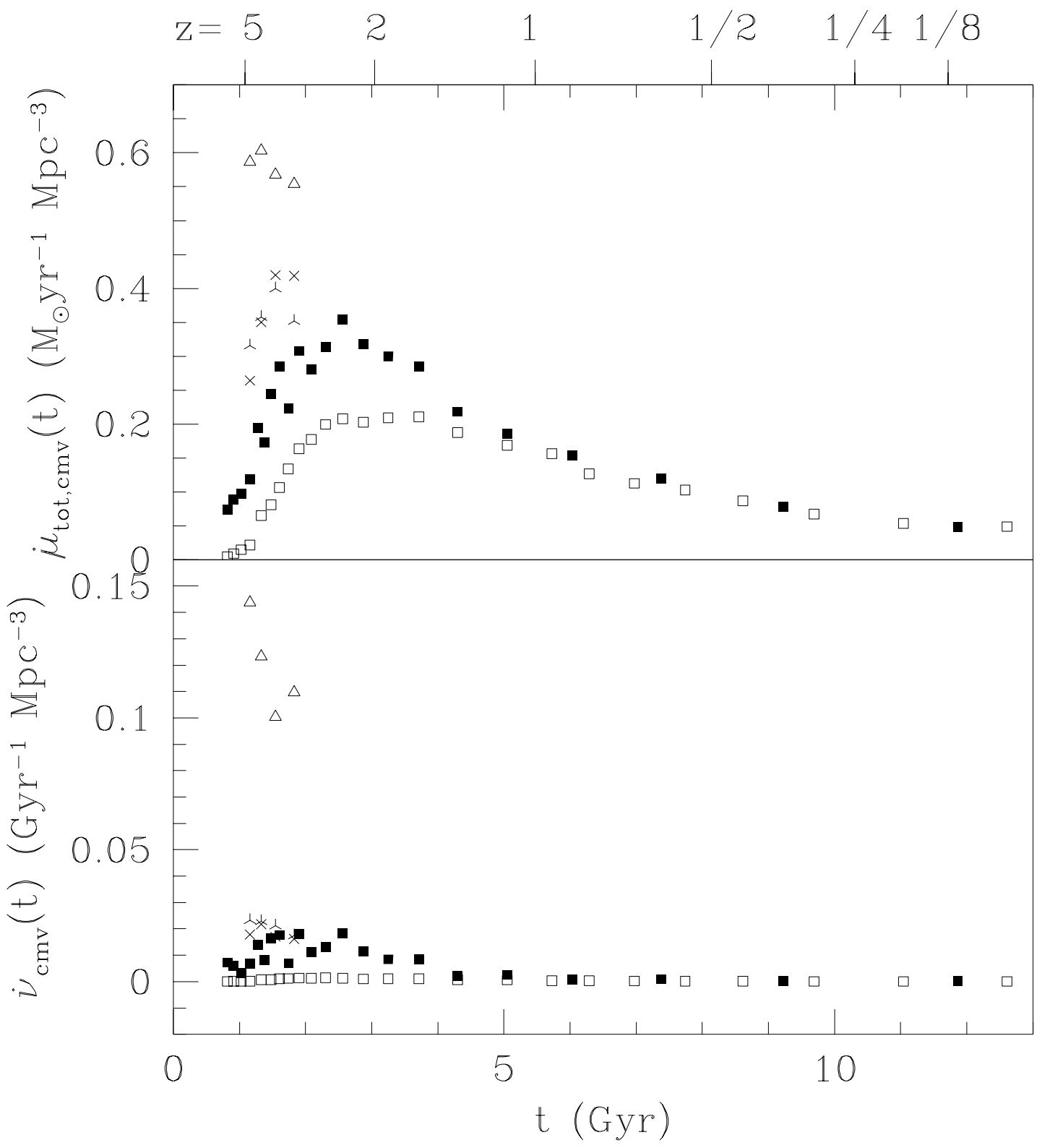

Fig. 3. - The rates of change of mass (top) and number density (bottom) above the masses shown in Figure 2. The number and mass densities for masses above $M_{c}=5.4 \times 10^{10} M_{\odot}$ (L50/144-open squares) and $M_{c}=6.8 \times 10^{9} M_{\odot}(\mathrm{L} 11 / 64$-solid squares $)$ agree for $z<1.5$. 
vey, the implied luminosity corresponding to the threshold $M_{c}$ of L50/144 is $\sim L_{*} / 4$, the precise value depending on whether we match the $g^{\prime}$ or $r^{\prime}$ luminosity function and whether we match space densities at $M_{c}$ or at $L_{*}$.

\section{Evolution of the galaxy population through accretion and mergers}

\subsection{Galaxy creation and destruction}

The instantaneous rate of change in galaxy number in the simulations is determined by the relative rates of formation and destruction. Galaxies are destroyed either through merging with another larger galaxy or though disruption. In the upper panel of Figure 4, open squares show the rate of change $\dot{\nu}$ of galaxy number density from the L50/144 simulation. (These are the same as the open squares in the lower panel of Figure 3, but the vertical scale is greatly expanded.) Filled squares show the creation rate of new galaxies; in general these galaxies are not entirely new, but they have gained enough mass since the last output to cross the mass threshold $M_{c}$. The difference between the filled and open points is the destruction rate, shown also by the filled squares in the lower panel. Open squares and filled triangles in this panel show the contributions to the destruction rate from mergers (which make one galaxy from two) and disruption (which moves a galaxy from above $M_{c}$ to below $M_{c}$ ), respectively. Figure 5 shows creation and destruction rates for $M_{c}=6.8 \times 10^{9} M_{\odot}$, from L11/64. This plot is noisier because of the smaller number of galaxies in the simulation, but it is qualitatively similar except for the higher number densities associated with less massive galaxies (note the change in vertical scale from Figure 4).

In both cases the creation rate climbs rapidly to a peak at $z \approx 3$ and declines steadily thereafter, though new galaxies continue to form (i.e., to cross the $M_{c}$ threshold) down to $z=0$. The merger rate climbs more gently, reaching a broad maximum at $z \sim 1-2$ and declining only slowly at lower redshift. Mergers always dominate over disruption as a destruction mechanism. At high redshift the creation rate is much larger than the destruction rate, but the balance begins to shift at $z<3$. By the present day, the creation and destruction rates are nearly equal, and both are much larger than the net rate. This result shows that a high galaxy merger rate need not lead to rapid evolution of the galaxy luminosity function, since other galaxies can grow to replace the ones that are lost.

\subsection{Growth of mass through accretion and mergers}

Figures 2 and 3 show that the total mass in resolved galaxies increases with time, as one would expect. Since we only count mergers among resolved objects, all new mass must enter the population through accretion. However, mergers redistribute mass within the resolved galaxy population, and we can sensibly ask whether existing resolved galaxies gain more of their mass through accretion or through merging with other resolved systems. Our methods for determining the smooth accretion 


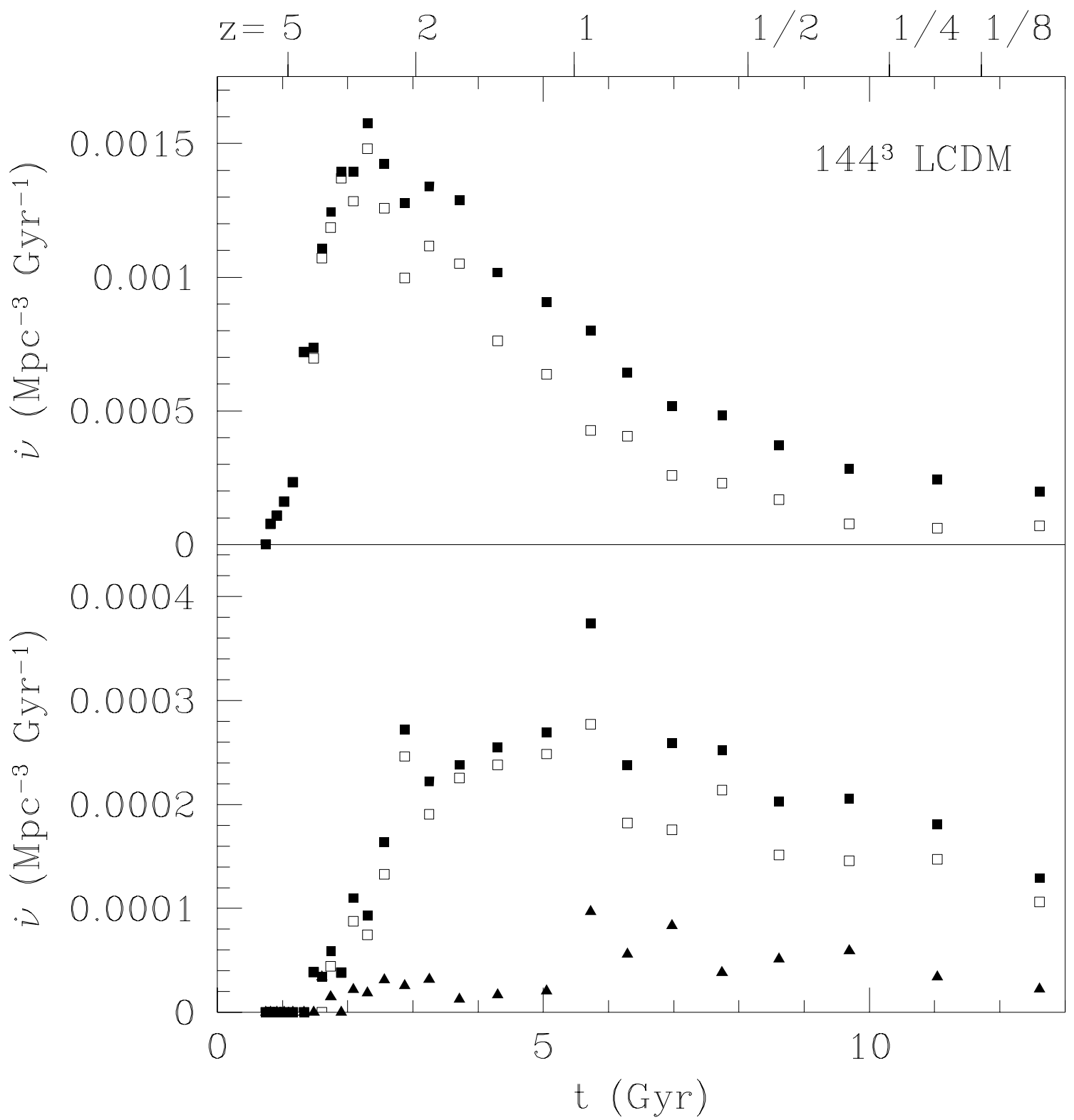

Fig. 4.- Contributions to the number density evolution for galaxies with masses above $M_{c}=$ $5.4 \times 10^{10} M_{\odot}$ (L50/144). Top panel: filled squares give the creation rate of new galaxies, and open squares give the net rate (creation minus destruction). Bottom panel: filled squares give the total destruction rate, and open squares and filled triangles show the respective contributions of mergers and disruption. 


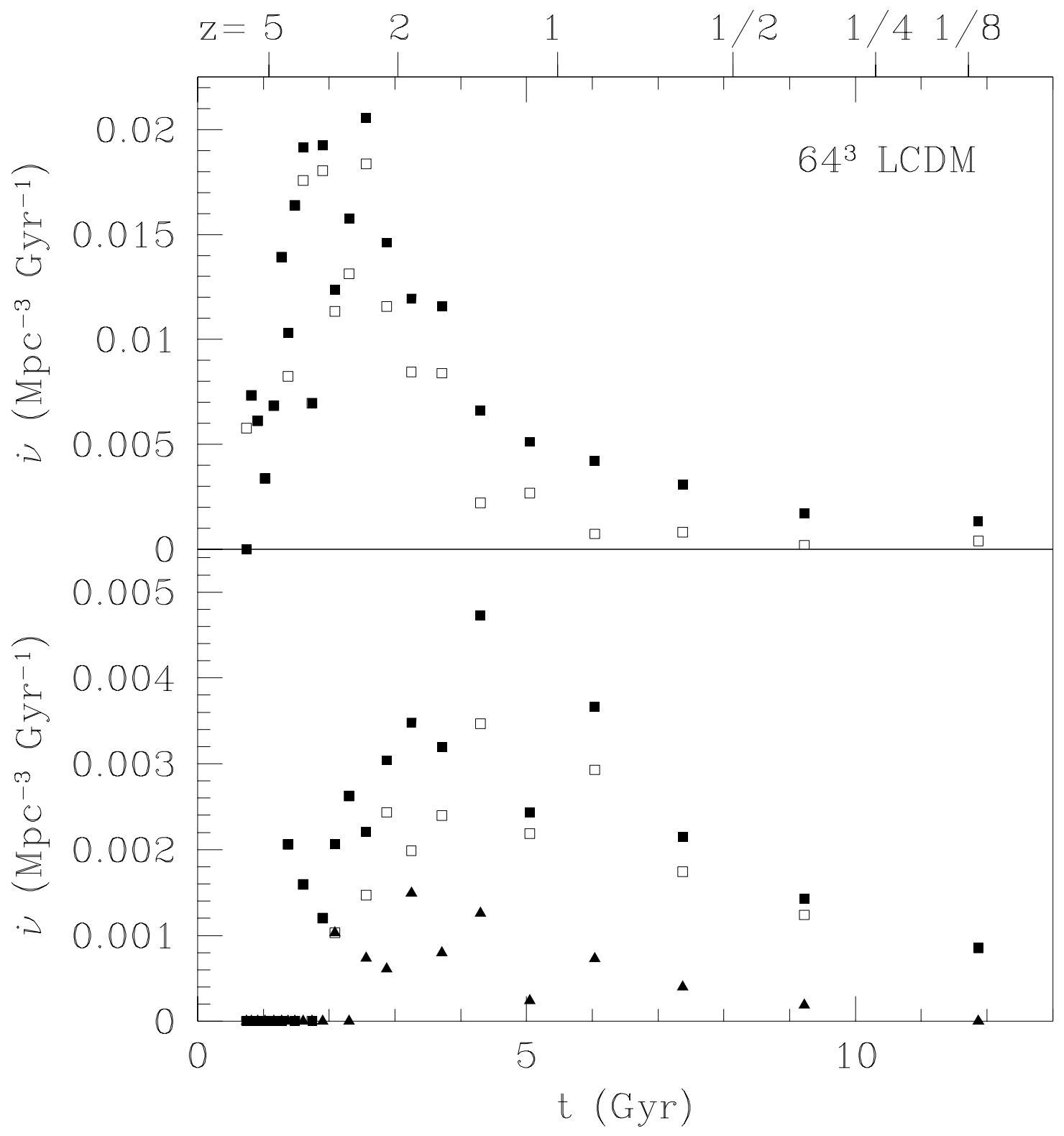

Fig. 5.- Same as Figure 4, for a mass threshold $M_{c}=6.8 \times 10^{9} M_{\odot}(\mathrm{L} 11 / 64)$. Note the change in vertical scale, reflecting the higher number density of the less massive galaxies. 
and merger rates from the simulation outputs are fully described in the Appendix. The most important point to recall here is that we define accretion to be true smooth accretion plus any merging with galaxies below the cutoff mass $M_{c}$, since we have no reliable way to distinguish these processes. For the remainder of this paper we will restrict our discussion to the L50/144 simulation because it has the largest dynamic range and probes the mass range of greatest interest.

In Figure 6, pentagons show the total mass accretion rate for galaxies with masses above $M_{c}=5.4 \times 10^{10} M_{\odot}$; except for the minor effects of disruption and mass loss, this is the same quantity shown by the open squares in Figure 3 (top panel). Since we would like to know how existing galaxies acquire mass, we subtract the contribution from newly formed galaxies and plot the remainder as the filled squares. This globally averaged mass accretion rate in galaxies above $5.4 \times 10^{10} M_{\odot}$ rises rapidly until $z \approx 2$ and declines slowly thereafter. At $z=0$, the accretion rate is about $1 / 3$ of its peak value. The rate of truly smooth accretion by galaxies above $M_{c}$ is necessarily lower than that shown in Figure 6, since higher resolution simulations would resolve some of this accretion into mergers with small groups. In addition to the total accretion rate, we show the contributions from accreted gas (open squares) and stars (crosses) separately. As expected, gas dominates the type of accreted material.

Figure 7 shows the distribution of accretion rates at four different redshifts. The mean mass accretion rate of resolved galaxies drops from $\dot{M} \approx 40 M_{\odot} \mathrm{yr}^{-1}$ at $z=1-2$ to $\dot{M} \approx 10 M_{\odot} \mathrm{yr}^{-1}$ at $z=0$, though at each redshift the $\dot{M}$ distribution is broad. Gas accretion always dominates over stellar accretion, as expected from the global properties in Figure 6.

Figure 8 shows the volume averaged rate at which resolved galaxies $\left(M>5.4 \times 10^{10} M_{\odot}\right.$ in L50/144) gain mass through merging with other resolved galaxies. The single most important result of this paper comes from the comparison of Figure 8 to Figure 6: galaxies gain most of their mass by accretion, not by mergers (note the large change in vertical scale). At $z \sim 2$, accretion dominates merging by about a factor of five. However, accretion declines more rapidly than merging towards low redshift, and by $z=0$ it dominates by only a factor of two. The ratio of accretion growth to merger growth in galaxies above this mass threshold would drop if our simulations had higher mass resolution and could therefore resolve objects that are currently counted in the smooth accretion rate. ${ }^{2}$ Nevertheless, the total merging rate is fully determined for galaxies with a mass above $M_{c}=5.4 \times 10^{10} M_{\odot}$, and in this mass range mergers contribute $\lesssim 1 / 3$ of the mass growth rate at every redshift. Furthermore, we will show in $\S 4.3$ that most of the merger contribution comes from relatively massive objects, so the overall accretion-to-merger ratio would remain high even with higher mass resolution (see discussion in $§ 5$ ).

Focusing on the merger rate itself, we see that it climbs fairly quickly until $z \sim 1.5$. The variations thereafter appear largely stochastic, with a slight overall decline. The clear decline in

\footnotetext{
${ }^{2}$ However, a higher resolution simulation would not necessarily give a lower accretion-to-merger ratio for its full population of resolved galaxies, since its mass resolution threshold would also be lower, and these less massive galaxies would still tend to accrete unresolved material.
} 


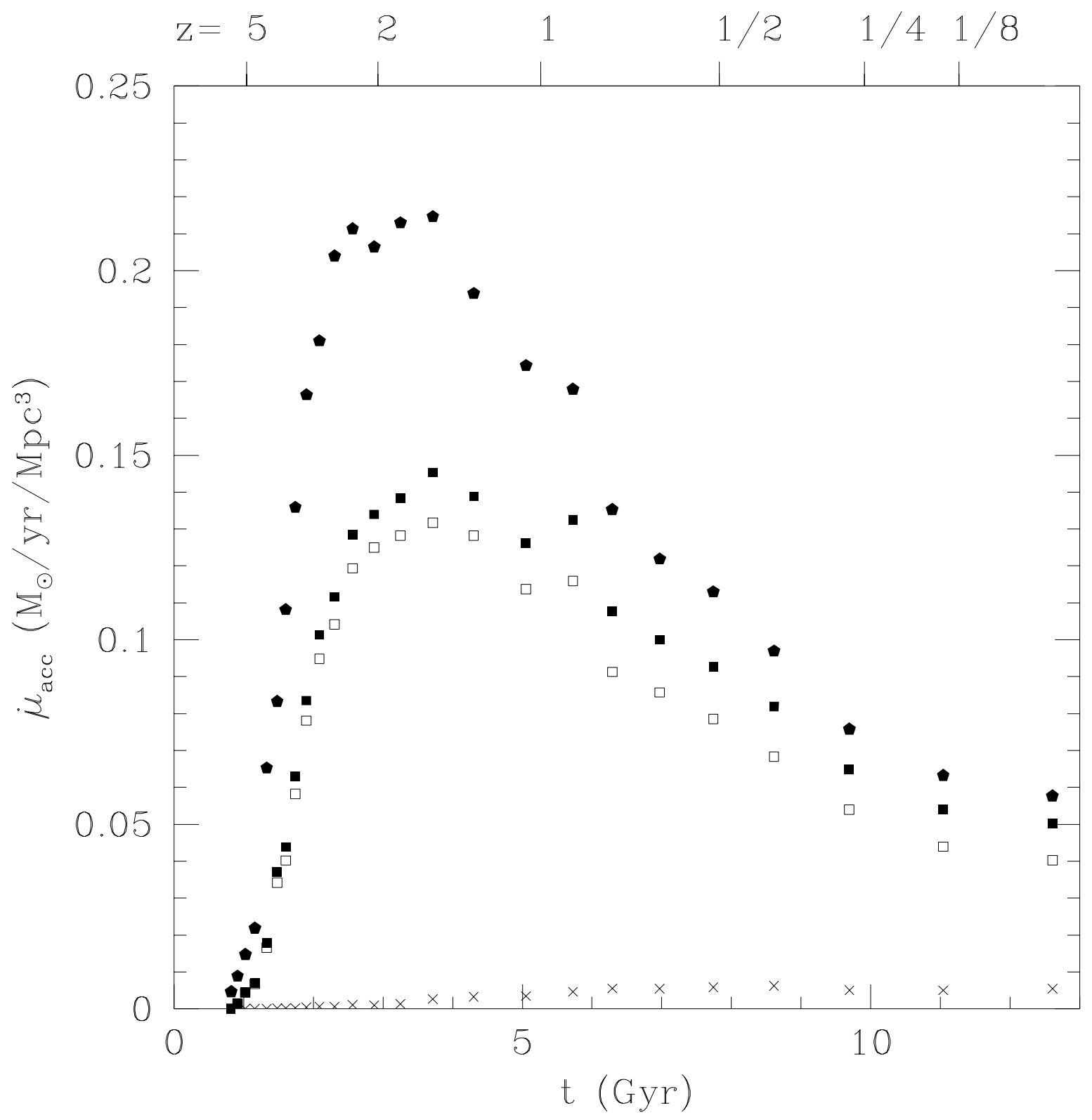

Fig. 6. - Evolution of the mass accretion rate for galaxies above $M_{c}=5.4 \times 10^{10} M_{\odot}(\mathrm{L} 50 / 144)$. solid pentagons show the total including the contribution from newly formed galaxies. Solid squares show only the accretion rate onto existing galaxies, excluding newly formed objects. Open squares show the contribution to this accretion from gas and crosses the contribution from stars. 


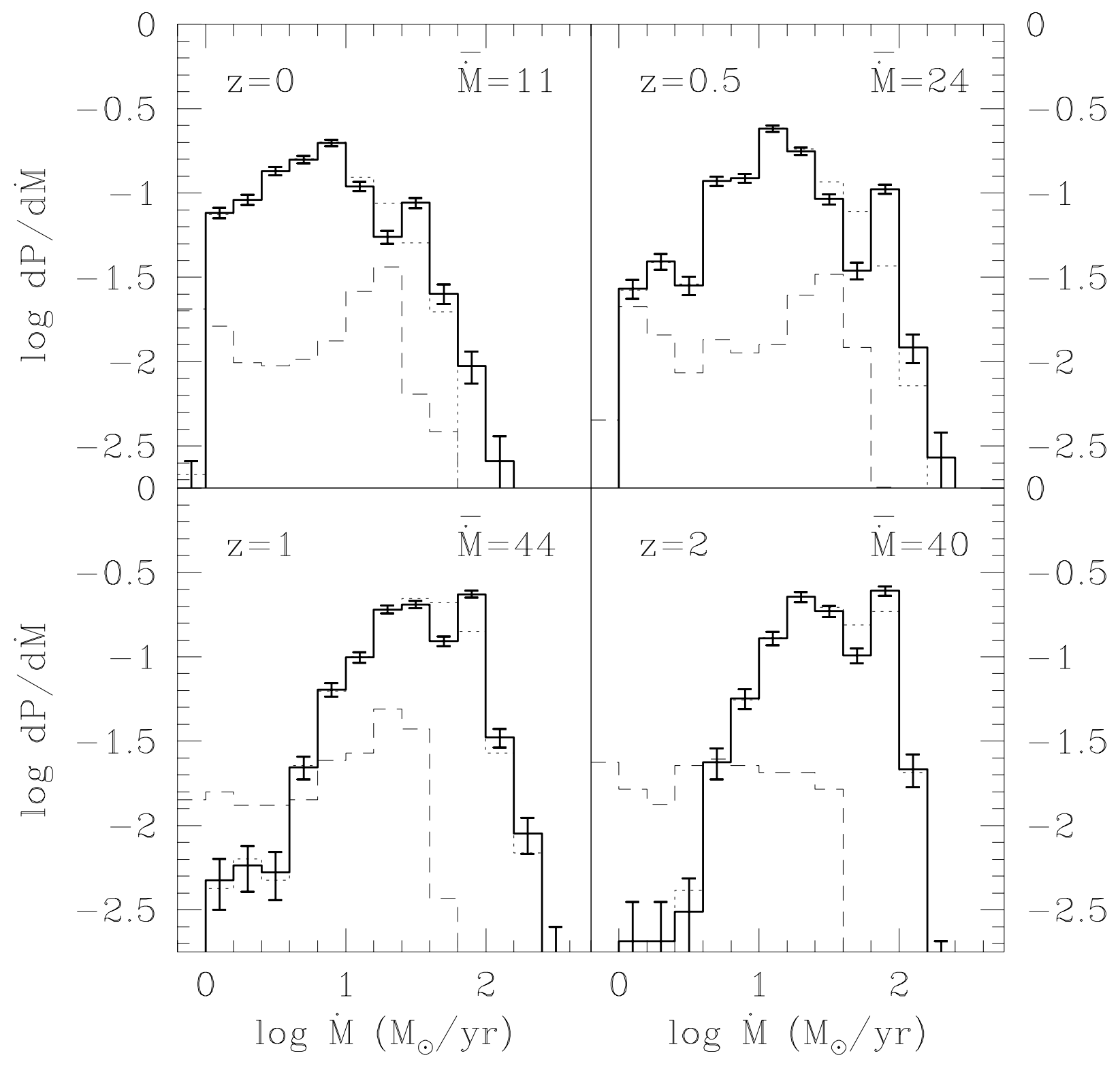

Fig. 7.- Distribution of accretion rates onto individual galaxies at different redshifts for galaxies with masses above $M_{c}=5.4 \times 10^{10} M_{\odot}($ L50/144). The solid line shows total accretion rates, the dotted line gas accretion rates, and the dashed line stellar accretion rates. The quantity $\overline{\dot{M}}$ denotes the mean accretion rate at each time. 


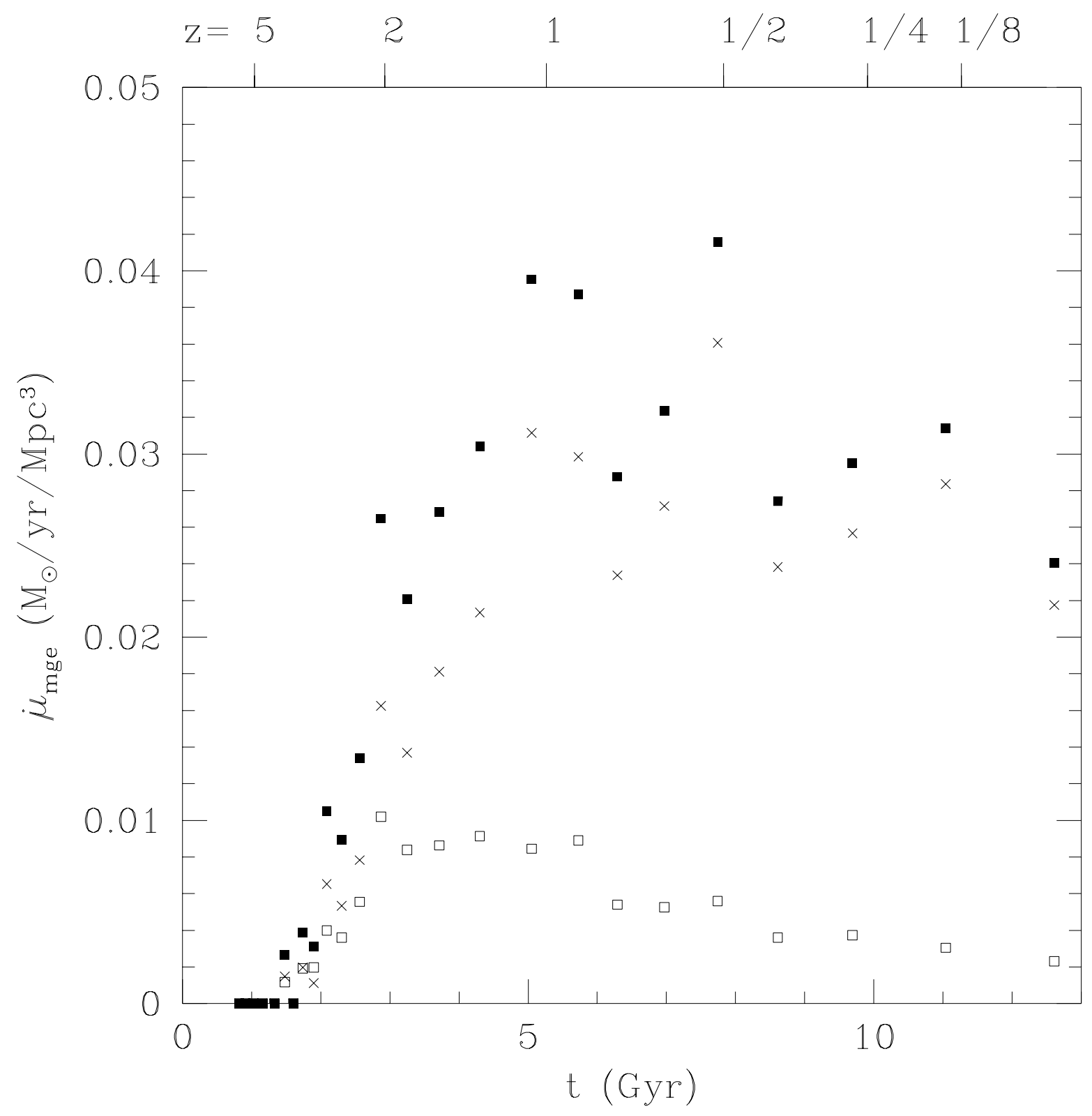

Fig. 8. - The rate of mass gain through merging as a function of time, for galaxies with masses above $M_{c}=5.4 \times 10^{10} M_{\odot}(\mathrm{L} 50 / 144)$. Solid squares show the total rate, open squares the contribution from gas, and crosses the contribution from stars. Note that the vertical scale is stretched by a factor of five compared to Figure 7. 
the number rate of merging (Figure 4) is compensated by an increase in the typical mass of the merging objects. Open squares and crosses in Figure 8 show the separate contributions of gas and stars. Gas-rich mergers are important early on, where about half the accreted material is gas and half is stars, but they are quickly overtaken by mergers with predominantly stellar systems.

\subsection{Masses and mass ratios of merging galaxies}

Figure 9 shows the probability distribution of the masses of smaller "satellite" galaxies that merge into larger "parent" galaxies. Above the cutoff mass, $M_{c}$, the distribution generally appears to follow a power law in mass. The slope of this power law decreases with redshift, presumably following the evolution of the non-linear mass scale. At high redshift $(z>0.5)$, the estimated slope is steep enough $(\alpha<-1)$ that small objects dominate in number, while large objects dominate in mass (i.e., an integral for the total mass of merging objects diverges at the upper end). However, at low redshift $(z<0.5)$, the slope flattens, so that large objects dominate in both number and mass.

Figure 10 shows the distribution of merger mass ratio, the ratio $f=M_{\text {sat }} / M_{\text {par }}$ between the satellite galaxy and the larger parent with which it merges. At the earliest times $(z>2)$, large mass ratios predominate, although the total amount of merging is somewhat smaller than at later times. Subsequently, a low $f$ tail develops, and the distribution becomes approximately scale free above the effective resolution cutoff. The probabilities extend to lower and lower $f$ as the simulation evolves. The slopes of the distributions are quite steep, with higher mass ratios $(f \gtrsim 0.4)$ dominating by number. However, it is important to keep in mind that our finite resolution suppresses low- $f$ mergers, and since the number of resolved galaxies is largest near $M_{c}$, there is some preference for merger ratio near unity almost by definition.

Given the resolution-limited mass, $M_{c}$, the simulation also gives an exact or complete estimate of the total amount of merging above a given mass ratio and corresponding parent mass. Stated in more mathematical terms, for a given merger ratio $f_{0}$, there is a corresponding parent mass, $M_{0}=M_{c} / f_{0}$, for which the simulation includes all mergers with $f \geq f_{0}$ and $M_{\text {par }} \geq M_{0}$. Conversely, given $f_{0}$, the rate is not exact for $M_{\text {par }}<M_{0}$ because the total should include satellites with $M_{\text {sat }}<M_{c}$, which are unresolved in the simulation.

Figure 11 shows the total number merger rate $\dot{\nu}\left(\geq M_{0}, \geq f_{0}\right)$ at different redshifts, while Figure 12 shows the corresponding mass merger rate $\dot{\mu}\left(\geq M_{0}, \geq f_{0}\right)$. Both figures display a range of values of $f_{0}$ and $M_{0}$. Of particular interest is the amount of major merging, since that could transform a galaxy's morphological type. We define a major merger to be $f \geq 0.25{ }^{3}$ With the present

\footnotetext{
${ }^{3}$ Simulations show that for $f \sim 1$, mergers essentially transform galaxy types (e.g. Barnes 1988, 1992; Hernquist 1992, 1993; Barnes \& Hernquist 1996), while for $f \sim 0.1$, galaxies are damaged, but not completely destroyed (e.g. Fullager et al. 1993; Walker et al. 1996).
} 


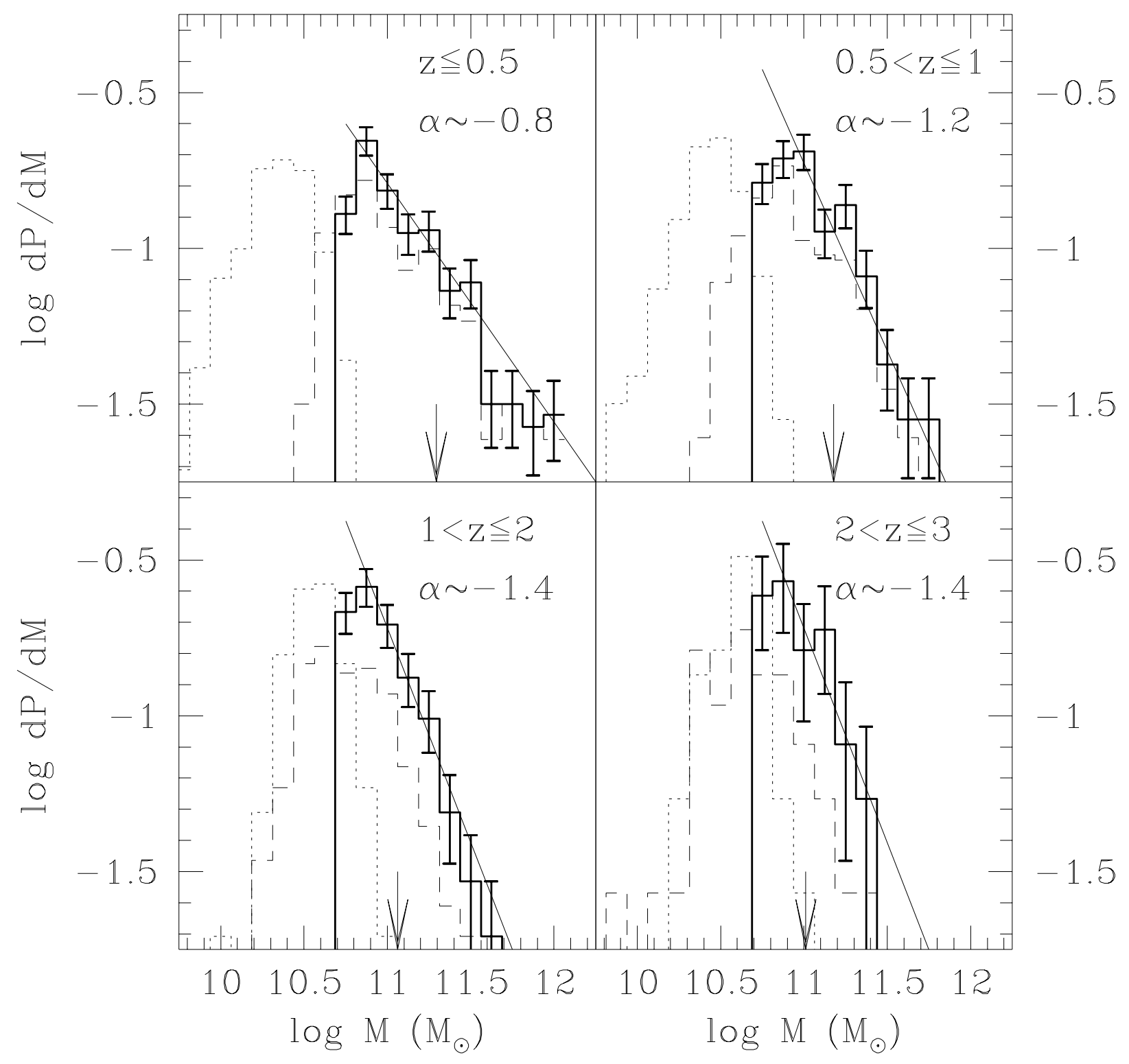

Fig. 9. - The mass distribution of galaxies that merge into larger galaxies as a function of smaller galaxy mass, in four redshift ranges, for galaxies with masses above $M_{c}=5.4 \times 10^{10} M_{\odot}(\mathrm{L} 50 / 144)$. The solid line shows total merged mass, the dotted line the gas mass, and the dashed line the stellar mass. There is a cutoff in total mass at $\log M=10.8$, corresponding to the minimum mass $M_{c}$, although stellar and gas contributions can be smaller. The line indicates the approximate slope $\alpha$ of the high-mass end. The arrow on the abscissa denotes the mean merger mass. 


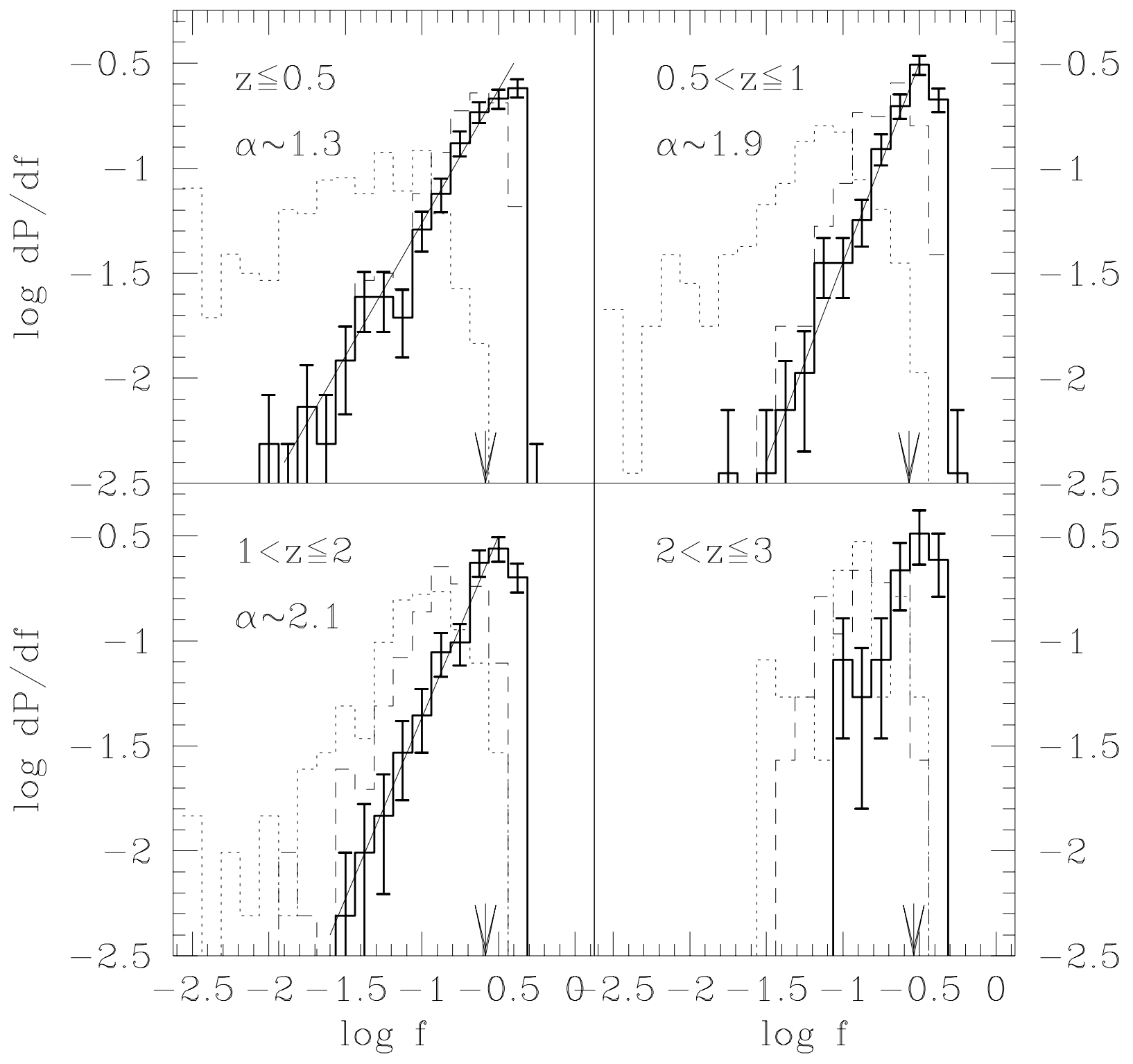

Fig. 10. - The fractional mass distribution of merger events into individual galaxies as a function of merger mass ratio, $f=M_{\text {sat }} / M_{\text {par }}$, in four different redshift ranges, for galaxies with masses above $M_{c}=5.4 \times 10^{10} M_{\odot}(\mathrm{L} 50 / 144)$. The solid line shows the distribution of total fractional mass, while the dotted and the dashed lines show the distribution of the gaseous and the stellar mass fraction, respectively. The line indicates the approximate slope $\alpha$ of the distribution. The arrow on the abscissa indicates the mean mass ratio of the merger, which is roughly $\bar{f}=0.25$ throughout. 
simulation, we can measure the amount of major merging exactly for $M_{\text {par }} \geq M_{0}=2.2 \times 10^{11} M_{\odot}$, approximately $L_{*}$. Overall, major merging appears to contribute significantly to the total amount of merging measured in the simulation. For this threshold mass at $z \sim 1,50 \%$ of the total mass in merging comes from pairs with $f_{0} \geq 0.25$. At $z=0$, the contribution increases to nearly $75 \%$. In terms of number, the rate lies in the range from $10^{-6}-10^{-5} \mathrm{Gyr}^{-1} \mathrm{Mpc}^{-3}$ for $z<3$. Figure 13 shows the rates for both number and mass as a function of redshift for major merging. For $z>3$, major merging does not occur at this resolution limit. For $z<3$, both rates climb. The number rate reaches a stochastically varying plateau for $z<2$, while the mass rate continues to climb until $z=1$, where it also reaches a plateau.

\subsection{Mass loss}

Fairly significant mass loss also occurs in the simulations. Figure 14 shows the mass lost from

galaxies as a function of time for galaxies with masses above $M_{c}=5.4 \times 10^{10} M_{\odot}(\mathrm{L} 50 / 144)$. The trend follows the merging history shown above - it rises until $z \sim 1$ and reaches a plateau for $z<1$. Overall, mass loss rates are about $25 \%$ of merging rates. Gaseous mass loss is nearly constant with time, while stellar mass loss increases with time. Stellar mass loss dominates after $z \sim 0.5$. Visual inspection shows that the mass loss occurs in highly clustered regions.

\subsection{Star formation}

The volume-averaged star-formation rate is closely related to the growth of galaxies in the simulation. Figure 15 plots the global star-formation rate $\dot{\mu}_{s f}$ for galaxies with masses above $M_{c}=5.4 \times 10^{10} M_{\odot}(\mathrm{L} 50 / 144)$ as a function of time. Interestingly, the overall rate (filled squares) closely follows the global rate of mass accretion shown in Figure 6 rather than the merging history shown in Figure 8. We also plot separately the star formation contributed by gas that is newly acquired through smooth accretion since the last time output and that contributed by gas already present within the parent galaxy or merged satellite in our last time slice (crosses and open squares, respectively). Most of the stars form from gas already present in galaxies. Moreover, since merging rates are much smaller than star-formation rates (c.f. Figures 6 and 8), this implies that star formation typically occurs in gas that was present in the parent galaxy at the previous timestep. Hence, in our simulations, gas that enters a galaxy typically waits for at least the interval between our outputs ( $1 / 3$ Gyr at high redshift and 4/3 Gyr at low redshift) before converting to stars.

\section{Discussion}

The results presented here describe the growth of the central baryonic components of galaxies

in the context of hierarchical structure formation. We have focused primarily on the relative 


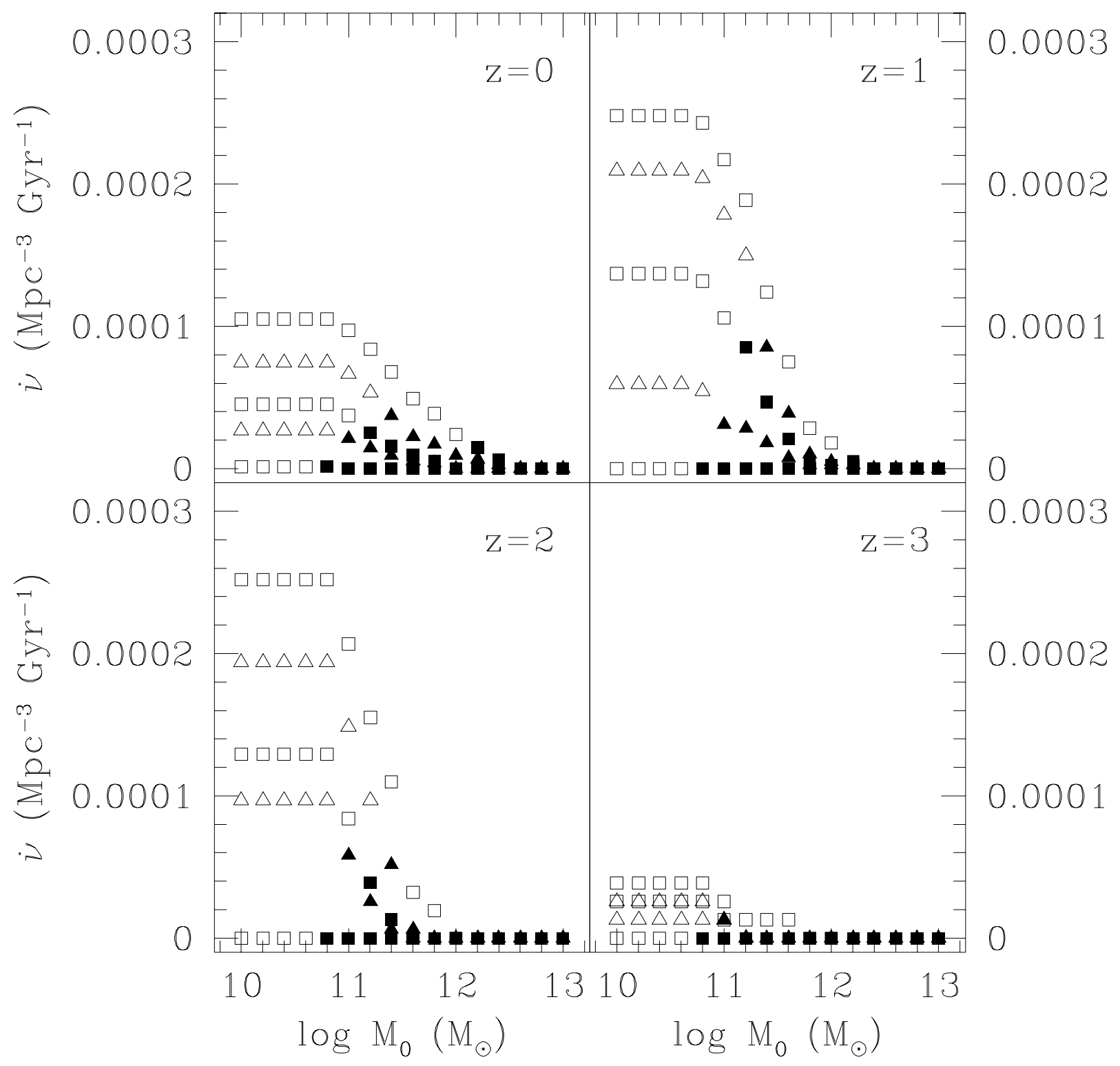

Fig. 11. - The cumulative number merger rate as a function of parent group mass for different merger ratios: $\dot{\nu}\left(\geq M_{0}, \geq f_{0}\right)$. Each sequence of points indicates a different $f_{0}$ : from top to bottom, $f_{0}=1 \%, 25 \%, 50 \%, 75 \%, 100 \%$. The solid symbols indicate the parent mass range for which $M_{s a t} \geq M_{c}$ for $f \geq f_{0}$, i.e. the mass of the merged object lies above the 64 -particle threshold. The open symbols indicate parent masses for which $M_{s a t}<M_{c}$. Alternating symbols are used for visual clarity. 


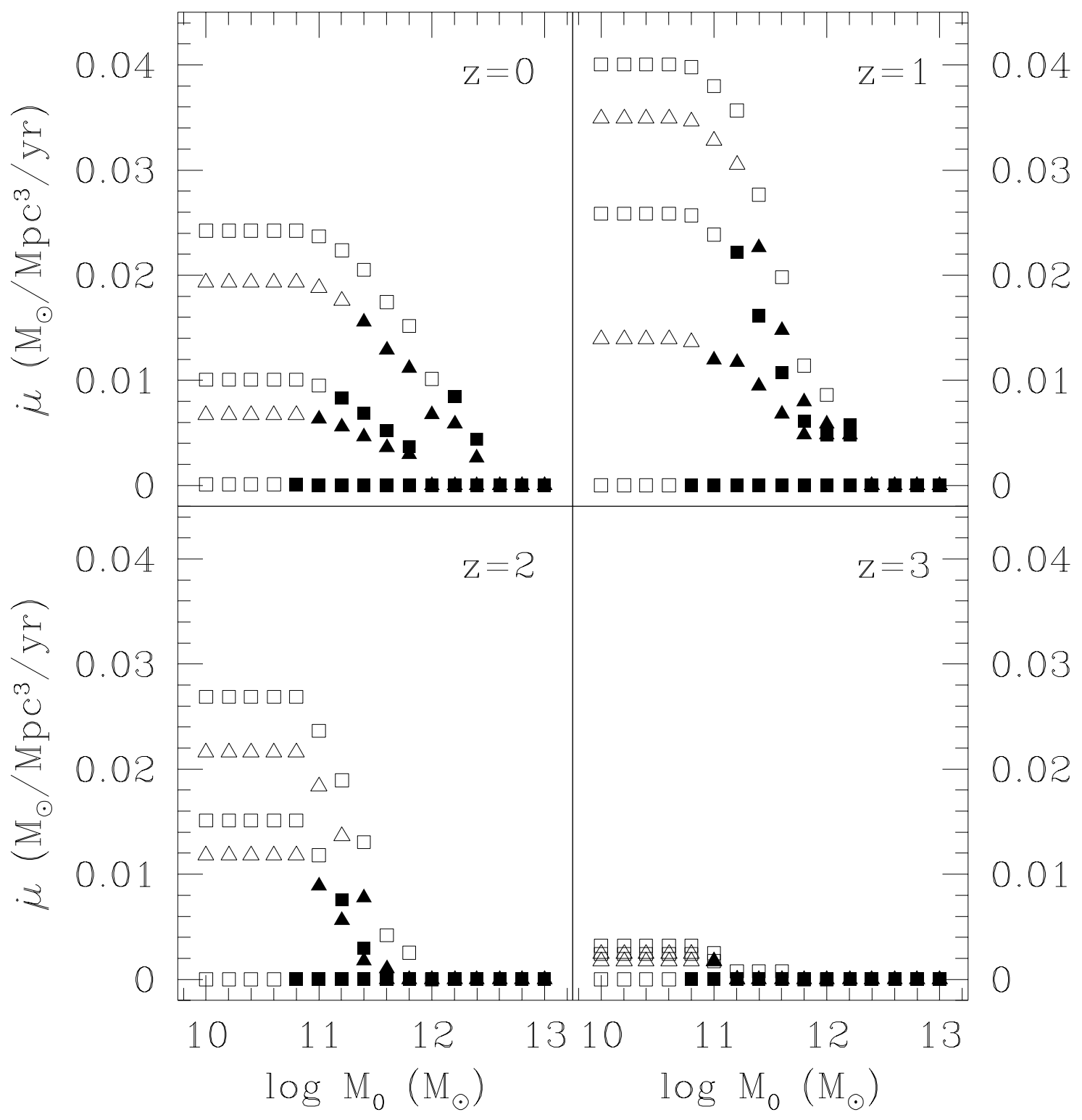

Fig. 12. - The cumulative mass merger rate as a function of parent group mass for different merger ratios: $\dot{\mu}\left(\geq M_{0}, \geq f_{0}\right)$. Each sequence of points indicates a different $f_{0}$ : from top to bottom, $f_{0}=1 \%, 25 \%, 50 \%, 75 \%, 100 \%$. The solid symbols indicate the parent mass range for which $M_{s a t} \geq M_{c}$. The open symbols indicate parent masses for which $M_{\text {sat }}<M_{c}$. Alternating symbols are used for visual clarity. 


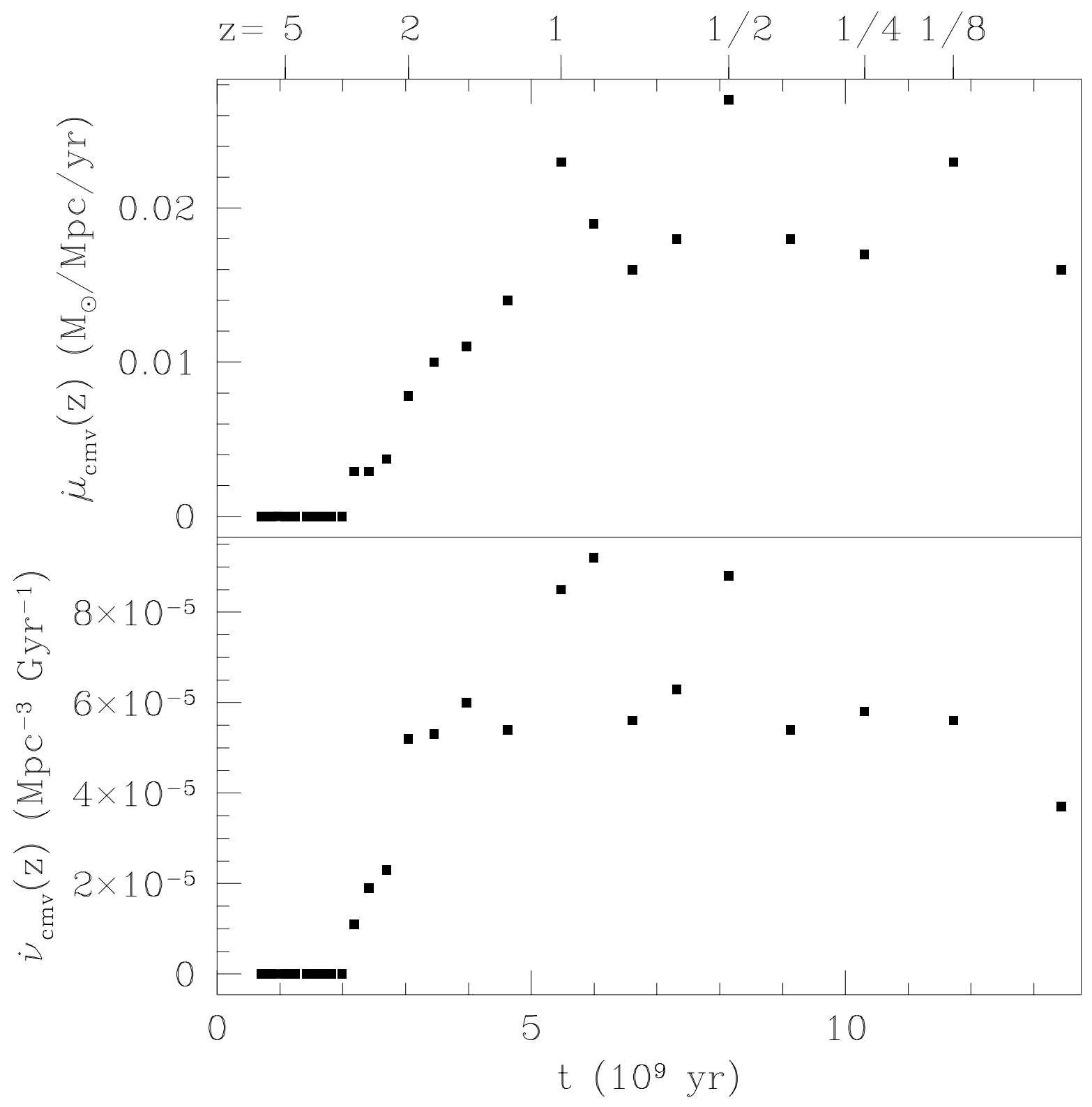

Fig. 13. - The evolution of number (bottom) and mass (top) merger rates with redshift for major mergers, $f_{0}=25 \%$ and $M_{0}=2.2 \times 10^{11} M_{\odot}$. 


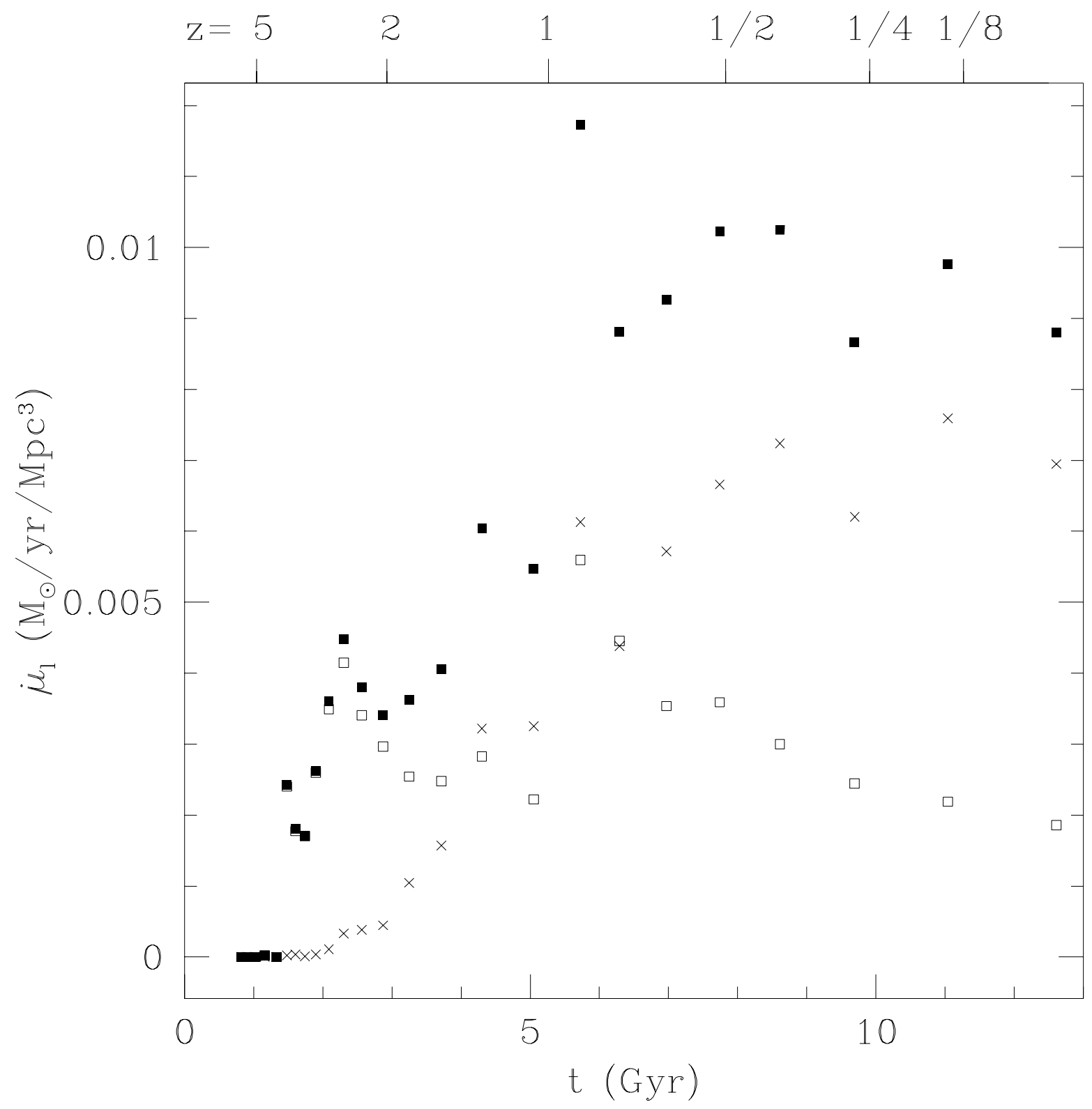

Fig. 14. - The rate of mass loss from existing groups per unit comoving volume as a function of time, for galaxies above $M_{c}=5.4 \times 10^{10} M_{\odot}$ (L50/144). Solid squares show the total, open squares the contribution from gas, and crosses the contribution from stars. 


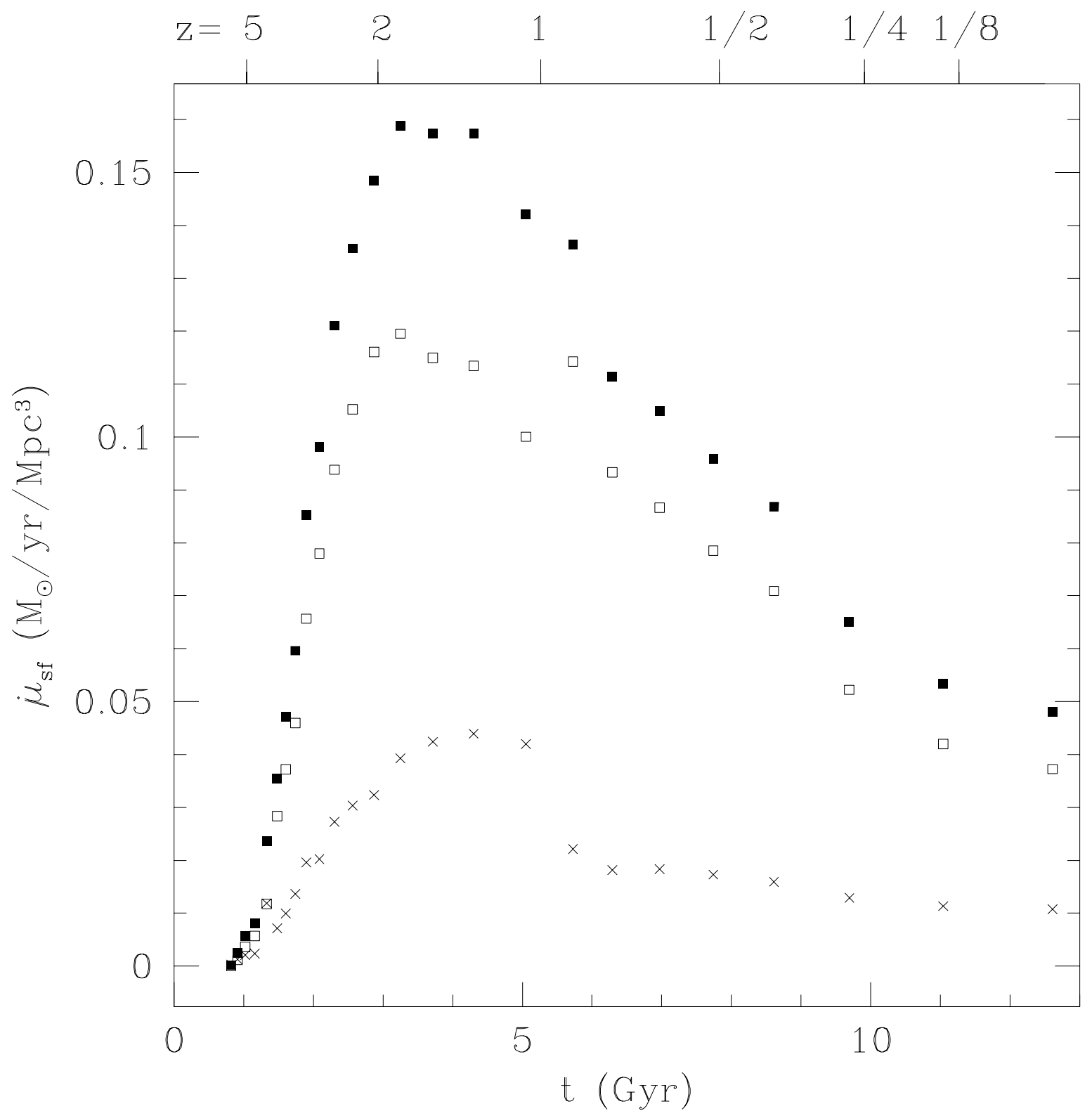

Fig. 15. - The star formation rate in galaxies above $M_{c}=5.4 \times 10^{10} M_{\odot}$ (L50/144) per unit volume as a function of time. The solid squares show the total, the open squares the contribution from gas already in groups and the crosses the contribution from gas that is recently accreted. 
contributions of merging and smooth accretion to the rate at which large galaxies gain mass. Overall we find that accretion dominates, especially at higher redshift, while merging becomes progressively more significant at later times.

The principal limitation of our results is that we cannot distinguish between smooth accretion and merging with objects below our mass resolution threshold, which is $M_{c}=5.4 \times 10^{10} M_{\odot}$ for our primary simulation (L50/144). As discussed at the end of $\S 3$, matching the observed galaxy space density implies that this mass threshold corresponds approximately to a luminosity $L_{*} / 4$. Our analysis gives well defined, and, we believe, numerically robust predictions for the merger rates of galaxies above this threshold with other galaxies above this threshold. These are lower limits to the merger rates of galaxies above this threshold with all other galaxies, and our corresponding accretion rates are upper limits to the rates of truly smooth accretion by these galaxies.

The mass spectrum of merging galaxies shown in Figure 9 gives useful guidance to the possible contribution of sub-resolution merging. Over roughly a decade in mass, the mass spectrum is approximately a power law $\propto M^{\alpha}$, with $\alpha \sim-0.8$ at $z=0$ and $\alpha \sim-1.4$ at $z=1-3$. The cumulative distribution of mergers above mass $M$ scales as $M^{\alpha+1}$, and the amount of mass contributed by mergers above mass $M$ scales as $M^{\alpha+2}$, thus diverging towards the high mass end of the spectrum. Thus, the measured slopes of the merger mass spectrum indicate that sub-resolution mergers should contribute relatively little mass compared to resolved mergers. To obtain a quantitative estimate, we assume that the measured power law mass spectrum extends from a high mass cutoff $M_{h}=6 \times 10^{11} M_{\odot}$ (see Figure 9 ) down to a low mass cutoff $M_{l}=0$. We then find that merging galaxies below $M_{c}$ should contribute roughly $25 \%$ of the total mass in mergers for $z>1$ $(\alpha \sim-1.4)$ and only about $5 \%$ of the total for $z<0.5(\alpha \sim-0.8)$. Thus, at high redshift, the simulation appears to account for more than $75 \%$ of the total mass in merging, whereas at low redshift it accounts for at least 95\%. Combined with the results in Figures 6 and 8, this implies that merging overall accounts for no more than $25 \%$ of the total mass in accretion and merging combined for $z>1$ and no more than $35 \%$ of the total for $z<0.5$. While higher resolution simulations will be needed to verify this estimate, the extrapolation from our current results implies that truly smooth accretion always exceeds merging by at least a factor of two in contributing to the mass evolution of galaxies.

It is interesting to compare the mass spectrum of mergers of baryonic objects found here to the mass spectrum of dark matter halo mergers, derived by Lacey \& Cole (1993) using the extended Press-Schechter formalism. The comparison is not exact because we determine the mass distribution of all merging objects, whereas Lacey \& Cole (1993) determine the distribution of objects falling into a single halo. The merger mass spectrum that they find is steeper than the one shown in our Figure 9. Nevertheless, the high redshift behavior is qualitatively similar in both cases: low mass objects dominate in number but high mass objects dominate in mass. At low redshift there is a qualitative difference in the two results: our scaling implies that high mass objects dominate in both mass and number, while Lacey \& Cole's implies that low mass objects still dominate in number but high mass objects dominate in mass. This difference could plausibly reflect the different dynamics 
of baryon and dark matter mergers. When two dark halos merge, the galaxies that they contain will not merge immediately. Dynamical friction can drag together massive galaxies more quickly than low mass galaxies, and this should make the merging mass spectrum of galaxies shallower than that of dark halos.

Our main point of contact with observations is through merging rates. Observationally, the efforts focus on determining the evolution of pair fraction with redshift (Zepf \& Koo 1989; Carlberg et al. 1994; Yee \& Ellingson 1995; Woods, Fahlman, \& Richer 1995; Patton et al. 1997; Abraham 1998; LeFèvre et al. 1999; Carlberg et al. 2000). The difficulty lies in identifying pairs of galaxies that should merge in a time of order the system dynamical time at increasingly large redshift. Here we use the true physical merger rates, since these are the quantities we have available from the simulations, but we do not investigate whether the observational analyses accurately infer these rates.

From their analysis of pairs selected from the CNOC2 and CFGRS surveys, Carlberg et al. (2000) have recently estimated the rate of mass growth due to merging in galaxies above about $0.2 L_{*}$ out to $z=1$. They find an integrated mass growth rate of about $2 \times 10^{-2} L_{*} \mathrm{Gyr}^{-1}$ per galaxy, with an estimated uncertainty of a factor of two. Their $0.2 L_{*}$ threshold should correspond approximately to our $M_{c}$ threshold, though there is a factor $\sim 2$ uncertainty in this identification. While Carlberg et al. (2000) do not explicitly present mass ratios for their pairs, it is likely that for such large galaxies the mergers are predominantly major $(f \geq 0.25)$. From Figure 13 we find that the major merging rate $\dot{\mu}_{m g e}$ at and above $M_{c}$ varies from $2 \times 10^{-2}$ to $3.5 \times 10^{-2} M_{\odot} \mathrm{Mpc}^{-3} \mathrm{yr}^{-1}$ between $z=1$ and $z=0$, while the comoving number density of objects varies from $4.2 \times 10^{-3}$ to $5.5 \times 10^{-3} \mathrm{Mpc}^{-3}$ over the same interval. Dividing the merger rate by the comoving number density and by an assumed value of $2.2 \times 10^{11} M_{\odot} L_{*}^{-1}$ yields rates of mass growth from major merging in the range $2 \times 10^{-2}-5 \times 10^{-2} L_{*} \mathrm{Gyr}^{-1}$ per galaxy. The agreement with the Carlberg et al. (2000) results is encouragingly good, given the theoretical and observational uncertainties that enter the comparison.

Another interesting feature of our results is the mass loss due to merging. Although the group finding algorithm introduces some uncertainty in the measurement, it does appear that a significant fraction of the mass in merging satellites winds up as intergalactic debris. Visual inspection of the simulations shows that this material permeates galaxy clusters and large galaxy groups and forms low-density halos around massive galaxies. Recent observational and theoretical work has provided evidence for the existence of such material, particularly in galaxy clusters (e.g. Theuns \& Warren 1997; Ciardullo et al. 1998; Ferguson, Tanvir \& von Hippel 1998; Calcanéo-Roldán et al. 2000). In future work, we will examine in more detail the properties of the debris produced by merging galaxies.

One obvious way to extend the work presented here is to employ new, higher resolution simulations. We are currently evolving a simulation similar to L11/64 but using $128^{3}$ particles in a $22.22 h^{-1} \mathrm{Mpc}$ box. This simulation will have larger dynamic range than L11/64 and greater 
overlap with L50/144, improving our ability to assess numerical resolution effects. In the longer term, a simulation with $288^{3}$ particles in a $50 h^{-1} \mathrm{Mpc}$ box would have the same mass resolution, $M_{c}=6.8 \times 10^{9} M_{\odot}$, as L11/64, sufficient to settle many of the remaining issues regarding the growth of $L_{*}$ galaxies in numerical simulations.

Motivated in part by the kinetic equation approach described in the Appendix, we have focused here on volume-averaged rates of accretion and mergers. These rates provide a good global view of activity in the resolved galaxy population, but there are other questions that can only be addressed by examining accretion and merger histories as a function of environment or galaxy by galaxy. For example, we would like to know what fraction of galaxies experience a major merger between $z=0.5$ and the present day, or what fraction have quiescent accretion histories from $z=1$ to $z=0$, and we would like to know how those fractions correlate with galaxy mass, environment, and stellar age. The analysis of mass acquisition can also be extended to examine how galaxies gain angular momentum or random kinetic energy. We will present results from such analyses in future work. Studies of this sort, applied to steadily improving numerical simulations, should provide a solid foundation for understanding the mass, luminosity, and morphological evolution of the galaxy population.

We thank Jeff Gardner for providing the basis for our merger code and for performing the L11/128 simulation. We also thank Mark Fardal, Enrico Vesperini and Martin Weinberg for helpful discussions. This work was supported by NASA Astrophysical Theory Grants NAG5-3922, NAG53820, and NAG5-3111, by NASA Long-Term Space Astrophysics Grant NAG5-3525, and by the NSF under grants ASC93-18185, ACI96-19019, and AST-9802568. The simulations were performed at the San Diego Supercomputer Center and NCSA.

\section{A. Evolutionary equation}

The simulations analyzed above can be viewed as Monte Carlo solutions of the kinetic equation that describes the evolution of the mass function $n(M, t)$ (and includes a large number of additional degrees of freedom, which we have projected over in our analysis). In the continuum limit, a simple form of the equation can be written:

$$
\begin{aligned}
\frac{\partial n}{\partial t} & =-\frac{\partial}{\partial M}[n \dot{M}]+\int_{M_{c}}^{M} d M^{\prime} n\left(M-M^{\prime}, t\right) n\left(M^{\prime}, t\right) \Gamma\left(M-M^{\prime}, M^{\prime}, t\right) \\
& -n(M, t) \int_{M_{c}}^{\infty} d M^{\prime} n\left(M^{\prime}, t\right) \Gamma\left(M, M^{\prime}, t\right)+C(M, t)-D(M, t) .
\end{aligned}
$$

On the right-hand side, the first term gives the rate of change of the baryonic mass $M$ of a galaxy

with accretion rate $\dot{M}$. Accretion gives rise to advective evolution in the mass phase space so the term is analogous to a continuity term. In writing this, we have assumed that the accretion $\dot{M}$ depends uniquely on the mass $M$, whereas, in reality, a sample of galaxies at mass $M$ would have 
a distribution of accretion rates. What is written here can be thought of as the evolution of the 'average' galaxy of mass $M$.

The second and third terms describe the merging of galaxies. The former gives the rate of creation of new galaxies of mass $M$ through the merging of pairs with masses $M^{\prime}$ and $M-M^{\prime}$ at the rate $\Gamma\left(M-M^{\prime}, M, t\right)$. The latter gives the loss rate of galaxies at $M$ through collisions with galaxies at $M^{\prime}$ at the rate $\Gamma\left(M, M^{\prime}, t\right)$. Note that we have ignored mass loss and disruption in writing these terms.

The fourth and fifth terms denote the creation and destruction of galaxies of mass $M$, respectively. In the simulation, galaxies are not created at all masses; instead they usually pass directly through the minimum mass threshold $M_{c}$ by mass accretion. In this case, one could introduce the creation rate as a boundary condition. Sometimes, however, sub- $M_{c}$ galaxies can merge and produce a new galaxy with a mass somewhat larger than $M_{c}$. As a result, we can qualitatively describe the creation function as a one-tail distribution that peaks at $M_{c}$ and has a fairly short tail to higher mass. Pure destruction does not really occur in the simulations at our adopted mass threshold, and the term has only been included for completeness.

By taking the first moment of equation (A1) with respect to $M$, one obtains the equation describing the evolution of the total baryonic mass in galaxies. From this, one can see that the total mass can change only through accretion onto and mass loss from existing galaxies and through creation and destruction. Merging, by contrast, alters only the number of galaxies.

\section{B. Changes in total mass}

It is straightforward to generalize equation (A1) to the discrete form that is required for analysis of the simulations. Assuming the discretized form of the equations, we write down the expressions relating the total accretion and mass loss to the change in the total amount of mass in galaxies between two times. Let there be $N_{1}$ groups at time $t_{1}$ and $N_{2}$ groups at time $t_{2}$ where $t_{2}>t_{1}$. Then the total mass in groups at either time $t_{k}$

$$
M_{k}=\sum_{i}^{N_{k}} m_{i},
$$

and the change in mass in groups between the two times

$$
\Delta M=M_{2}-M_{1}=M_{a c c}-M_{l o s s},
$$

where $M_{a c c}$ is the mass accreted smoothly and $M_{\text {loss }}$ is the mass lost from the progenitor groups at time $t_{1}$. Here we have included mass growth from created galaxies in the accretion term and mass loss from destroyed galaxies in the loss term. Defining $f_{i}$ as the fraction of group $i$ at $t_{1}$ that 
contributes to some group at $t_{2}$, we can write

$$
M_{\text {loss }}=\sum_{i}^{N_{1}}\left(1-f_{i}\right) m_{i}
$$

So that

$$
M_{a c c}=M_{2}-\left(M_{1}-M_{\text {loss }}\right)=M_{2}-\sum_{i}^{N_{1}} f_{i} m_{i} \equiv M_{2}-M_{g r p},
$$

where $M_{g r p}$ denotes the group mass at $t_{1}$ that contributes to the group mass at $t_{2}$. Using $M_{g r p}$, we can define the merged mass

$$
M_{\text {merge }}=M_{g r p}-\sum_{j}^{N_{2}} f_{i_{\max } j} m_{i_{\max } j}
$$

where $f_{i_{\max } j}$ and $m_{i_{\max } j}$ denote the fraction and total mass of the largest group at $t_{1}$ that contributes to group $j$ at $t_{2}$.

\section{Changes in gas/star mass}

The expressions given above change when considering individual gas and star components because of star formation. In this case, defining $M_{i}$ as the total gas mass in $N_{i}$ groups at time $t_{i}$, we can write the total change in gas mass in groups:

$$
\Delta M^{g}=M_{2}^{g}-M_{1}^{g}=M_{a c c}^{g}+M_{s}^{g}-M_{l o s s}^{g}-M_{g}^{s}
$$

where $M_{a c c}$ denotes the amount of gas accreted into groups, $M_{s}^{g}$ the gas mass created from stars, $M_{\text {loss }}^{g}$ the amount of gas lost from pre-existing groups, and $M_{g}^{s}$ the stellar mass created from gas. Of course, in the present simulations there is no stellar evolution mass loss so $M_{s}^{g}=0$. As in the definition above, $M_{\text {loss }}^{g}=\sum_{i}\left(1-f_{i}\right) m_{i}^{g}$, the respective sum of the fraction of mass in groups at $t_{1}$ that does not remain in groups at $t_{2}$. For stars, the treatment is analogous; however the star formation terms change sign.

To determine the $M_{g}^{s}$ term, we must consider the gas and star masses of the individual particles at consecutive outputs. However, because of the finite time resolution, it is impossible to say

whether star formation occurs before or after material is added to the new system. Here we assume that star formation occurs after material is added to a new system at time $t_{2}$.

With the total particle mass at time $t_{1} \mu_{k, 1}=\mu_{k, 1}^{g}\left(t_{1}\right)+\mu_{k, 1}^{s}$, the total stellar mass in groups at $t_{1}$ that contribute to the total mass in groups at $t_{2}$ is

$$
M_{g r p}^{s}\left(t_{1}\right)=\sum_{i}^{N_{1}} f_{i} m_{i}^{s}=\sum_{i}^{N_{1}} f_{i} \sum_{k}^{n_{i}} \mu_{i, k, 1}^{s} .
$$


However, the stellar mass of any particle may differ at $t_{2}$, so that $M_{g r p}^{s}\left(t_{2}\right) \neq M_{g r p}^{s}\left(t_{1}\right)$. The difference is, of course, related to the net gain or loss due to star formation and stellar evolution mass loss:

$$
M_{g}^{s}-M_{s}^{g}=M_{g r p}^{s}\left(t_{2}\right)-M_{g r p}^{s}\left(t_{1}\right) .
$$

Similarly, the change in gas mass implies that

$$
M_{s}^{g}-M_{g}^{s}=M_{g r p}^{g}\left(t_{2}\right)-M_{g r p}^{g}\left(t_{1}\right) .
$$

Since there is no stellar evolution mass loss, $M_{s}^{g}=0$. The treatment for accreted matter is analogous.

\section{REFERENCES}

Abraham, R. 1998 in IAU 186, Galaxy interactions at low and high redshifts, astro-ph/9802033

Barnes, J. 1988, ApJ, 331, 699

Barnes, J. 1992, ApJ, 393, 484

Barnes, J. \& Hernquist, L. 1992, ARA\&A, 30, 705

Barnes, J. \& Hernquist, L. 1992, ApJ, 471, 115

Barnes, J.E., \& Hut, P. 1986, Nature, 324, 446

Blanton, M.R. et al. 2001, AJ, 121, 2358

Burles, S., \& Tytler, D. 1997, AJ, 114, 1330

Burles, S., \& Tytler, D. 1998, ApJ, 499, 699

Calcanéo-Roldán, C., Moore, B., Bland-Hawthorn, J., Malin, D. \& Sadler, E. M. 2000, MNRAS, 314,324

Carlberg, R., Pritchet, C. \& Infante, L. 1994, ApJ, 435, 540

Carlberg, R. G., Cohen, J., Patton, D. R., Blandford, R., Hogg, D. W., Yee, H. K. C., Morris, S. L., Lin, H., Hall, P. B., Sawicki, M., Wirth, G. D., Cowie, L. L., Hu, E. \& Songaila, A., 2000, ApJ, 532, L1

Ciardullo, R., Jacoby, G. H., Feldmeier, J. J. \& Bartlett, R. E. 1998, ApJ, 492, 62

Davé, R., Dubinski, J., \& Hernquist, L. 1997, New Astron, 2, 227

Davé, R., Katz, N., Hernquist, L., \& Weinberg, D.H. 2001, in preparation.

Evrard, A.E., Summers, F.J., \& Davis, M. 1994, ApJ, 422, 11 
Ferguson, H., Tanvir, N. \& von Hippel, T. 1998, Nature, 391, 461

Fullager, D., Quinn, P.J. \& Hernquist, L. 1993, ApJ, 403, 74

Gelb, J. \& Bertschinger, E. 1994, ApJ, 436, 467

Gingold, R.A. \& Monaghan, J.J. 1977, MNRAS, 181, 375

Hernquist, L. 1987, ApJS, 64, 715

Hernquist, L. 1992, ApJ, 400, 460

Hernquist, L. 1993, ApJ, 409, 548

Hernquist, L. \& Katz, N. 1989, ApJS, 70, 419

Hernquist, L., Katz, N. \& Weinberg, D. H. 1995, ApJ, 442, 57

Hu, W. \& Sugiyama, N. 1996, ApJ, 471, 542

Katz, N., Hernquist, L., \& Weinberg, D. H. 1992, ApJ, 399, L109

Katz, N., Hernquist, L., \& Weinberg, D. H. 1999, ApJ, 523, 463

Katz, N., Weinberg D.H., \& Hernquist, L. 1996, ApJS, 105, 19 (KWH)

Lacey, C. \& Cole, S. 1993, MNRAS, 262, 627

Le Fèvre, O., Abraham, R., Lilly, S. J., Ellis, R. S., Brinchmann, J., Schade, D., Tresse, L., Colless, M., Crampton, D., Glazebrook, K., Hammer, F., Broadhurst, T. 2000, MNRAS, 311, 565

Lucy, L. 1977, AJ, 82, 1013

Moore, B., Quinn, T., Governato, F., Stadel, J. \& Lake, G. 1999, MNRAS, 310, 1147

Navarro, J. \& Steinmetz, M. 2000, ApJ, 528, 607

Patton, D., Pritchet, C., Yee, H., Ellingson, E. \& Carlberg, R. 1997, ApJ, 475, 29

Quinn, T., Katz, N., Stadel, J. \& Lake, G. 1997, astro-ph/9710043

Rauch, M., Miralda-Escude, J., Sargent, W.L.W., Barlow, T.A., Weinberg, D.H., Hernquist, L., Katz, N., Cen, R. \& Ostriker, J.P. 1997, ApJ, 489, 7

Seljak, U., \& Zaldarriaga, M. 1996, ApJ, 469, 437

Schechter, P. 1976, ApJ, 203, 297

Sheth, R. K., \& Tormen, G. 2001, MNRAS, submitted, astro-ph/0105113 
Spergel, D. \& Steinhardt, P. 2000, PRL, 84, 3760

Stadel, J., Katz, N., Weinberg, D.H., \& Hernquist, L. 2001, in preparation

Theuns, T. \& Warren, S. 1997, MNRAS, 284, L11

Toomre, A. \& Toomre, J. 1972, ApJ, 178, 623

Toth, G. \& Ostriker, J. P. 1992, ApJ, 389, 5

Walker, I., Mihos, J.C. \& Hernquist, L. 1996, ApJ, 460, 121

Weinberg, D. H., Davé, R., Gardner, J., Hernquist, L. \& Katz, N. 1999, in Photometric Redshifts and High-Redshift Galaxies, eds. R. Weymann, L. Storrie-Lombardi, M. Sawicki \& R. Brunner, (San Francisco: ASP Conference Series), 341, astro-ph/9908133

Weinberg, D. H., Hernquist, L., \& Katz, N. 1997, ApJ, 477, 8

Weinberg, D. H., Hernquist, L., \& Katz, N. 2000, ApJ, submitted, astro-ph/0005340

White, S. D. M., Efstathiou, G. P., \& Frenk, C. S. 1993, MNRAS, 262, 1023

Woods, D., Fahlman, G. \& Richer, H. 1995, ApJ, 454, 32

Yee, H. \& Ellingson, E. 1995, ApJ, 445, 37

Zaldarriaga, M., Seljak, U., \& Bertschinger, E. 1998, ApJ, 494, 491

Zepf, S. \& Koo, D. 1989, ApJ, 337, 34 